ARTICLE

Check for updates

https://doi.org/10.1038/s41467-021-22517-1

\title{
Osteocyte transcriptome mapping identifies a molecular landscape controlling skeletal homeostasis and susceptibility to skeletal disease
}

Scott E. Youlten (10 1,2, John P. Kemp (10 ${ }^{3,4}$, John G. Logan ${ }^{5}$, Elena J. Ghirardello ${ }^{5}$, Claudio M. Sergio (1) ${ }^{1}$, Michael R. G. Dack ${ }^{5}$, Siobhan E. Guilfoyle ${ }^{5}$, Victoria D. Leitch (10 5,6, Natalie C. Butterfield (1) ${ }^{5}$, Davide Komla-Ebri (1) ${ }^{5}$, Ryan C. Chai ${ }^{1}$, Alexander P. Corr (1) 1,2,7, James T. Smith (1) 1,2,7, Sindhu T. Mohanty (1) ${ }^{1}$, John A. Morris (1) 8,9, Michelle M. McDonald ${ }^{1,2}$, Julian M. W. Quinn (1) ${ }^{1}$, Amelia R. McGlade', Nenad Bartonicek ${ }^{10}$, Matt Jansson ${ }^{11,12}$, Konstantinos Hatzikotoulas (10) ${ }^{13,14}$, Melita D. Irving ${ }^{15}$, Ana Beleza-Meireles ${ }^{16}$, Fernando Rivadeneira (1) 17, Emma Duncan (10) 18,19,20, J. Brent Richards (1) 18,21, David J. Adams (1) 22, Christopher J. Lelliott (10) 22, Robert Brink 2,23, Tri Giang Phan (10) 2,23, John A. Eisman 1,2,24,

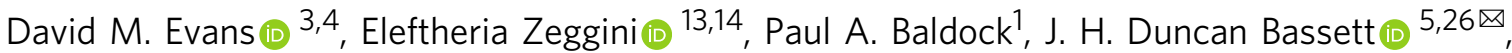
Graham R. Williams (i) ${ }^{5,26 \bowtie}$ \& Peter I. Croucher (1) 1,2,25,26凶

Osteocytes are master regulators of the skeleton. We mapped the transcriptome of osteocytes from different skeletal sites, across age and sexes in mice to reveal genes and molecular programs that control this complex cellular-network. We define an osteocyte transcriptome signature of 1239 genes that distinguishes osteocytes from other cells. 77\% have no previously known role in the skeleton and are enriched for genes regulating neuronal network formation, suggesting this programme is important in osteocyte communication. We evaluated 19 skeletal parameters in 733 knockout mouse lines and reveal 26 osteocyte transcriptome signature genes that control bone structure and function. We showed osteocyte transcriptome signature genes are enriched for human orthologs that cause monogenic skeletal disorders $\left(P=2.4 \times 10^{-22}\right)$ and are associated with the polygenic diseases osteoporosis $\left(P=1.8 \times 10^{-13}\right)$ and osteoarthritis $\left(P=1.6 \times 10^{-7}\right)$. Thus, we reveal the molecular landscape that regulates osteocyte network formation and function and establish the importance of osteocytes in human skeletal disease. 
T he skeleton is a highly dynamic structure that changes in shape and composition throughout life. Osteocytes are the most abundant cell type in bone and have emerged as master regulators of the skeleton. These enigmatic cells are connected via ramifying dendritic processes that form a complex multicellular network distributed throughout mineralised bone $\mathrm{e}^{1,2}$. The scale and complexity of the osteocyte network is comparable to neurons in the brain, with 42 billion osteocytes present in the human skeleton forming 23 trillion connections $\mathrm{s}^{2,3}$. This network enables osteocytes to detect and respond to mechanical strain, hormones and local growth factors and cytokines ${ }^{1}$. The network responds by regulating the formation and activity of osteoclasts and osteoblasts, instructing these cells to repair damaged bone, controlling bone mass and composition, and ensuring the optimal distribution of bone tissue in response to mechanical stress. Osteocytes also remove and replace bone surrounding the osteocyte network, a surface area of $>200 \mathrm{~m}^{2,3}$, by the process of perilacunar remodelling, liberating calcium and phosphate in response to endocrine demands ${ }^{4}$. These features allow the osteocyte network to maintain both the structural integrity of the skeleton and mineral homeostasis. Osteocytes also have regulatory functions beyond the skeleton, including in skeletal muscle, adipose tissue, the central nervous system and in the control of phosphate homeostasis and energy expenditure, indicating the network acts as an important endocrine organ ${ }^{5-8}$.

Although osteocytes are pivotal in controlling the skeleton, the molecular programmes that regulate their formation and function are poorly defined. Osteocytes are entombed within bone making them challenging to isolate and study. As a result osteocytes have been omitted from large-scale efforts to map tissue-specific transcriptomes ${ }^{9-12}$ and studies of their transcriptome are limited ${ }^{13-16}$. Consequently, the influences of anatomical location, age and sex on osteocyte regulatory pathways are unclear and their role in the pathogenesis of skeletal diseases is unknown.

Mutations in genes that have highly enriched expression in osteocytes cause rare bone and mineral disorders. For example, autosomal recessive inactivating mutations in SOST, which encodes the Wnt-antagonist sclerostin, result in the high bone mass disorder sclerosteosis type 1 (OMIM 269500) ${ }^{17}$. Deletion of a SOST regulatory element causes van Buchem disease (OMIM $239100)^{18,19}$ and inactivating mutations in $D M P 1$, the gene encoding dentin matrix acidic phosphoprotein 1, cause autosomal recessive hypophosphataemia (OMIM 241520) ${ }^{20}$. However, despite the classification of monogenic skeletal disorders identifying over six hundred individual diseases ${ }^{21}$, beyond a limited number of exceptions the role of genes enriched in osteocytes in these disorders remains largely unknown.

Common, complex skeletal diseases, including osteoporosis and osteoarthritis (OA), are also highly heritable. Genetic factors contribute to $50-80 \%$ of the variance in bone mineral density (BMD), the major determinant of osteoporosis susceptibility, and account for $>50 \%$ of the variance in susceptibility to $\mathrm{OA}^{22-24}$. Nevertheless, recent large-scale genome-wide association studies (GWAS) have defined only a proportion of the heritability in $\mathrm{BMD}$ and $\mathrm{OA}$ susceptibility ${ }^{25,26}$, underscoring the need for alternative approaches to identify genes contributing to these diseases. We hypothesised that mutations in genes with enriched expression in osteocytes would cause rare monogenic skeletal dysplasias and mineral disorders, and contribute to the risk of common polygenic skeletal diseases including osteoporosis and osteoarthritis.

To address this hypothesis, we developed a method to define a transcriptomic map of the osteocyte network and used it to investigate relationships between genes with enriched expression in osteocytes and human skeletal disease. We defined the genes expressed in osteocytes isolated from different skeletal sites, at different ages and from both sexes, and discovered bone-specific and sexually dimorphic differences in the osteocyte transcriptome during post-natal development. We defined an osteocyte transcriptome signature, a profile of genes with enriched expression in osteocytes, and discovered novel genes and molecular programmes that control formation and function of the osteocyte network. Finally, we showed that osteocyte transcriptome signature genes are (i) highly enriched for genes that cause monogenic skeletal disorders and (ii) significantly over-represented with susceptibility genes for BMD and OA identified by human GWAS.

\section{Results}

The osteocyte-enriched transcriptome is highly conserved throughout the skeleton. To investigate the genes that control osteocytes, we first identified the repertoire of genes expressed in osteocytes, the osteocyte-enriched transcriptome, from three different skeletal sites (Fig. 1a, b). Total RNA was isolated from skeletal samples (98\% cortical bone, $2 \%$ cancellous bone) highly enriched for osteocytes ( $>80 \%$ of cells present) from the tibiae, femora and humeri of 16-week-old mice $(n=8)$ and sequenced (Fig. 1b, c and Supplementary Fig 1a-d). A threshold of 'active' gene expression was determined based on the distribution of normalised gene expression in each sample ${ }^{27}$ (Fig. 1d and Supplementary Data 1).

In all, 14,794 genes were actively expressed in the three bone types with $92 \%$ of genes expressed at all sites and $96 \%$ of genes validated in one of three independent datasets, including the IDG-SW3 osteocytic cell-line, laser-capture micro-dissected osteocytes or osteocytes isolated by collagenase digestion (Fig. 1e, f and Supplementary Data 2). These comprised protein-coding genes, long non-coding RNAs (lncRNAs), genes present in the GENCODE transcriptome annotation but yet to be experimentally confirmed (TEC), and novel genes yet to be reported in any public annotation (Fig. 1e). The number of genes actively expressed in osteocytes was similar to other tissues, including 8997 genes expressed in all 12 organs and tissues examined ${ }^{28}$ (Supplementary Fig. 2a and Supplementary Data 2). Osteocytes formed a separate cluster in a principal component analysis (PCA), confirming the osteocyte-enriched transcriptome is clearly distinct from the transcriptome of other tissues (Supplementary Fig. 2b). In support of this, analysis of gene expression specificity, using the Tau measure ${ }^{29}$ calculated across these tissues, showed a significant proportion of genes with high specificity of expression for osteocytes (Supplementary Fig. 2c).

Whilst the osteocyte-enriched transcriptome was conserved among bones, 27 genes were differentially expressed between skeletal sites (LFC $>0.5, P<0.05$, Fig. $1 \mathrm{~g}$ and Supplementary Data 3). These included seven genes expressed specifically in either the fore (two genes) or hindlimb (five genes) (Fig. 1h). All encoded developmental transcription factors. They included Tbox $5(T b \times 5)$ and homeobox-d9 (Hoxd9), expressed exclusively in the humerus and known to establish forelimb identity ${ }^{30,31}$. By contrast, Homeobox-c8-c11 (Hoxc8, Hoxc9, Hoxc10 and Hoxc11) and paired-like homeodomain 1 (Pitxl) were expressed in the femur and tibia only, and not the humerus. In the developing limb bud, Pitxl is expressed exclusively in the hindlimb and is the master regulator of hindlimb-type identity ${ }^{32}$. Aberrant expression of PITX1 in the forelimb leads to homeotic arm-to-leg transformation in Liebenberg syndrome (OMIM 186550) ${ }^{33}$. Fifty-two percent of the 27 differentially expressed genes, were homeobox genes, or Hox-antisense lncRNAs, indicating this family may be important in maintaining the identity of osteocytes at different sites (Fig. 1h). Together these data show that the osteocyte transcriptome is highly conserved at different 
a

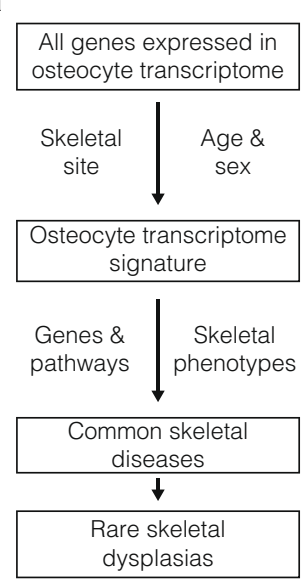

b

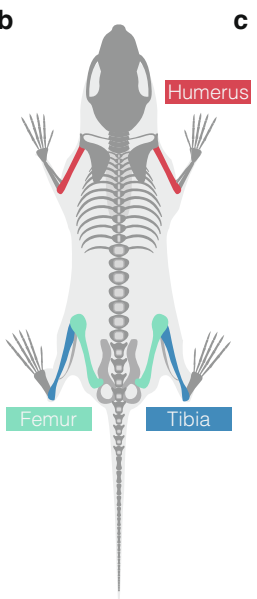

c i

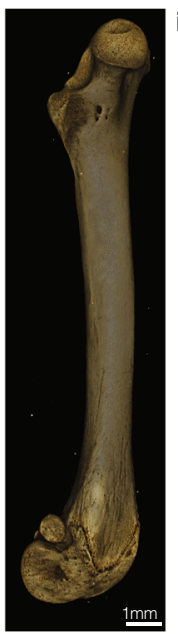

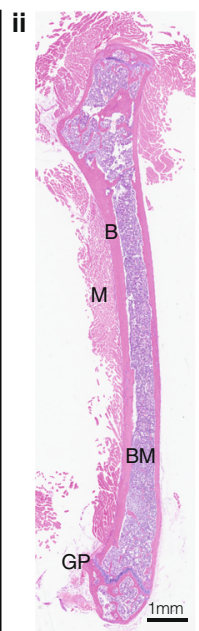
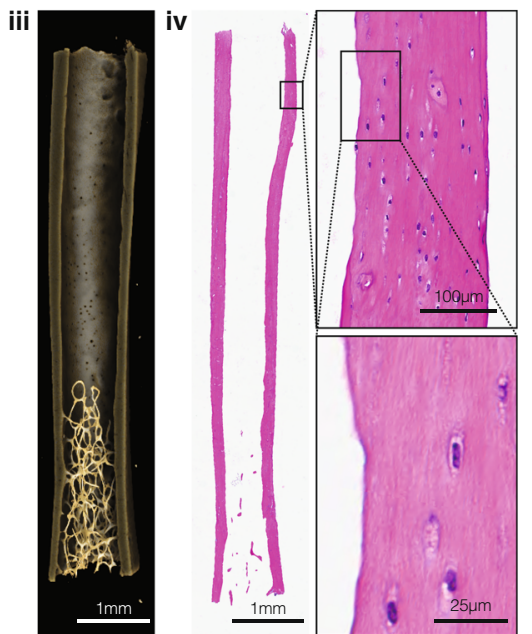

d i Replicate specific threshold

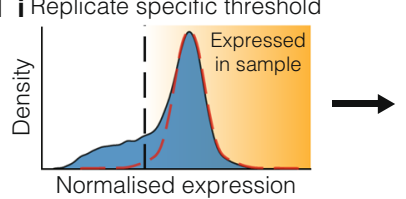

ii Gene activity

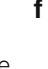

e i

\begin{tabular}{|c|c|c|c|c|c|}
\hline Biotype & Tibia & Femur & Humerus & Any bone & All bones (\%) \\
\hline Protein & 12675 & 12657 & 12847 & 13072 & $12394(95)$ \\
\hline IncRNAs & 849 & 858 & 866 & 1016 & 706 (69) \\
\hline TEC & 486 & 485 & 496 & 531 & 447 (84) \\
\hline Novel & 72 & 75 & 75 & 103 & $49(48)$ \\
\hline Other & 56 & 55 & 51 & 72 & $39(54)$ \\
\hline Total & 14138 & 14130 & 14335 & 14794 & 13635(92) \\
\hline
\end{tabular}

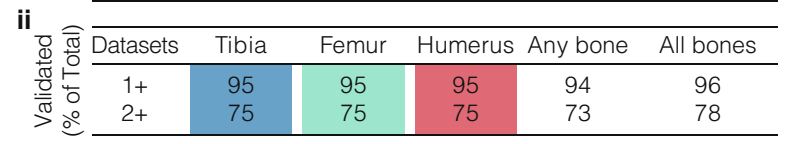

g

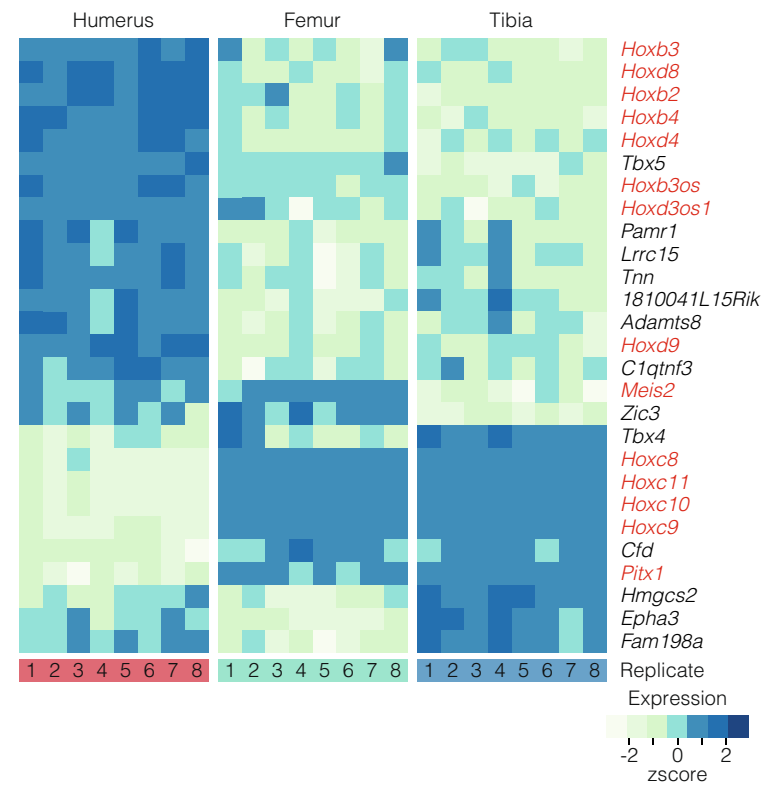

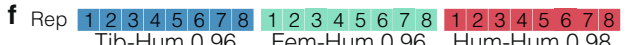
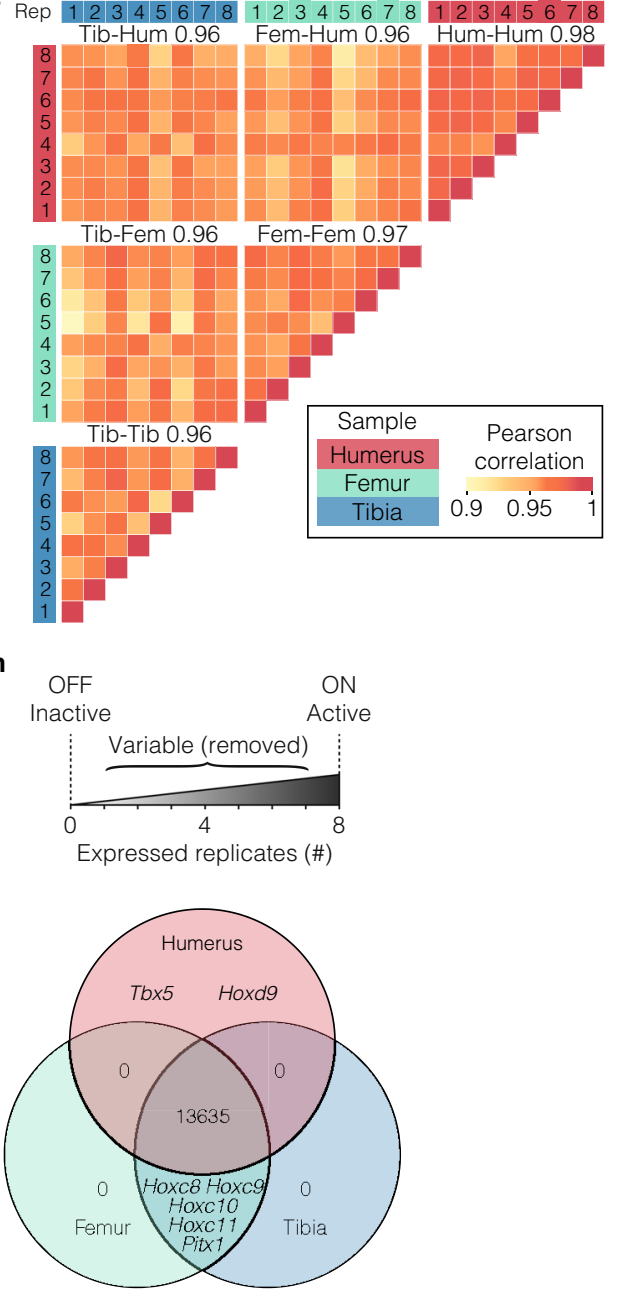

anatomical sites, although, homeobox genes, which are typically associated with patterning in development, identify osteocytes from different skeletal locations, even in adult mice.

Sex and age differences in the osteocyte-enriched transcriptome. Since bone structure and bone mass vary with sex and age $^{34-36}$, we hypothesised that the osteocyte-enriched transcriptome differs between the sexes and changes with age. We therefore analysed bone structure and the transcriptome of male and female mice at different ages (Fig. 2a). Bone length and bone mineral content (BMC), but not bone mineral density (BMD), differed between sexes, whereas bone length, BMC and BMD increased with age in both sexes (Fig. $2 \mathrm{~b}$ and Supplementary Fig. 3). The total number of genes actively expressed by osteocytes 
Fig. 1 The osteocyte transcriptome is broadly conserved across the skeleton. a Schematic of the overall study design used to define the osteocyte transcriptome, to define an osteocyte transcriptome signature, the profile of genes enriched in osteocytes, and to identify their role in skeletal disease. b Diagram illustrating the 3 bone-types in which the osteocyte transcriptome was sequenced and compared. c Representative micro-CT and histological images of bone samples prior to processing (i-ii) and following osteocyte isolation (iii-iv), illustrating the effective removal of bone marrow, muscle and growth plates and enrichment of osteocytes (iv) ( $n=8$ biological independent mice). Boxes and dotted lines identify areas of high magnification confirming osteocyte isolation. Bone (B), bone marrow (BM), muscle (M) and growth plate (GP) are identified. $\mathbf{d}$ Actively expressed genes were identified based on the normalised gene expression distribution (blue histogram) and a hypothetical distribution of active promoters (red dotted line) ${ }^{27}$ (i). Vertical line denotes the sample-specific threshold of actively expressed genes. Genes were considered active if they were above sample-specific threshold in all replicates, inactive if they were below the threshold in all replicates, or variable if they were above the threshold in at least one but not all replicates (ii). e The number of active genes and biotype composition in the osteocyte transcriptome across skeletal sites (i), and the percentage of total active genes detected in osteocytes in at least one (1+) and two or more (2+) orthogonal datasets (ii). The Any bone column reflects the number of genes actively expressed at any skeletal site. The All bones column reflects the number and proportion (as a percentage) of genes that were actively expressed in all skeletal sites. TEC $=$ To be Experimentally Confirmed. $\mathbf{f}$ Pearson correlation of gene expression between individual replicates and bone types (mean of each bone type comparison represented by numbers above heatmap). Tib = tibia, Fem = femur, Hum = humerus, Rep = biological replicate. $\mathbf{g}$ Genes that were differentially expressed between osteocytes isolated from different bone types. Homeobox genes and antisense-RNAs are in red (FDR $\leq 0.05, \mathrm{LFC}>0.5$ ). h Methodology for identifying genes expressed in a site-specific manner and a Venn diagram identifying the genes expressed in one bone type but not others.

increased with skeletal maturation with $81 \%$ of genes expressed at all ages in both sexes (Fig. 2c and Supplementary Data 2). Comparison of gene expression between age groups showed differences between 4 and 10 weeks of age (female $P=8 \times 10^{-3}$, male $P=3 \times 10^{-5}$ ), but not from 10 to 16 weeks or $16-26$ weeks, demonstrating the osteocyte transcriptome expressed during growth is distinct from the transcriptome in the adult skeleton (Fig. 2d). Comparison of the osteocyte transcriptome between sexes showed it was different between male and female mice in the mature skeleton ( 16 weeks, $P=8.0 \times 10^{-3}$ and 26 weeks, $P=$ $2.8 \times 10^{-2}$ ), but not at earlier ages (Fig. $2 \mathrm{e}$ ).

To identify genes and processes that contribute to differences in age and sex, clusters of co-expressed genes were identified by weighted gene co-expression network analysis. Seven clusters of correlated genes (denoted by seven colours) were identified (Supplementary Fig. 4a and Supplementary Data 4). One cluster (denoted by Grey) contained genes that were not correlated with each other or other clusters. Between $86 \%$ and $97 \%$ of genes in each cluster were also found in at least one of three orthogonal datasets, including the IDG-SW3 osteocytic cell line, micro-dissected osteocytes or osteocytes isolated by collagenase digestion (Supplementary Fig. 4b). Each cluster was associated with distinct biological processes (Supplementary Fig. 4b). The expression of genes within two clusters (purple and turquoise) increased with age, whereas, gene expression in two clusters (black and brown) decreased with age (Supplementary Fig. 4a). The expression of genes in the Brown and Magenta clusters were associated with both age and sex (Supplementary Fig. 4a and Supplementary Fig. 5a). The Brown cluster contained genes encoding bone matrix constituents, including osteocalcin (Bglap, Bglap2), osteonectin (Sparc) and bone sialoprotein (Ibsp) and was associated with proteinprocessing and transport (Supplementary Fig. 4a and Supplementary Data 4). The Magenta cluster included cathepsin $\mathrm{K}$ (Ctsk), tartrate resistant acid phosphatase (Acp5) and the vacuolar ATPase family and was associated with processes relating to bone resorption (GO:0045453, $P=4.37 \times 10^{-6}$ ), osteoclast differentiation (GO:0030316, $P=1.40 \times 10^{-8}$ ), $\mathrm{pH}$ reduction (GO:0045851, $P=2.31 \times 10^{-6}$ ) and ATP-coupled cation transport (GO:0099132, $P=2.13 \times 10^{-14}$; Supplementary Fig 5b, c and Supplementary Data 4). To exclude a contribution to the Magenta cluster from genes expressed in contaminating osteoclasts we stained paraffin sections of osteocyte-enriched bone samples for tartrate resistant acid phosphatase (TRAP) and confirmed the absence of TRAP positive osteoclasts on trabecular and cortical endosteal bone surfaces (Supplementary Fig 5d). Furthermore, analysis of the top 20 Magenta cluster genes, which include genes typically found in osteoclasts, showed that 19 were found in at least one of the three orthogonal datasets (Supplementary Fig 5e). Together this suggests the Magenta cluster genes may be important in regulation of perilacunar-resorption ${ }^{15,37}$. To investigate further, we examined expression of Magenta cluster genes in lactating mice in a publicly available microarray dataset $^{15}$. Sixty-six of the 84 Magenta genes were up-regulated during lactation $\left(P=3.8 \times 10^{-35}\right)$ and this was reversed postlactation $\left(P=1.2 \times 10^{-15}\right.$; Supplementary Fig $\left.5 f\right)$ strengthening the notion that the Magenta cluster identifies genes involved in perilacunar remodelling. Together, this highlights the dynamic regulation of the osteocyte transcriptome during post-natal skeletal development and identifies clusters of genes that are differentially regulated during skeletal maturation and between the sexes.

A unique transcriptome signature defines the osteocyte. To identify the genes that distinguish osteocytes specifically, we identified a profile of genes whose expression was enriched in osteocytes relative to other cell types. We hypothesised that genes important for osteocyte-specific functions are actively expressed and preferentially expressed in osteocytes compared to other cell lineages within bone, including bone marrow cells and cells lining bone (Fig. 3a). To test this, we performed transcriptome analysis on bone samples enriched with osteocytes, from which bone marrow and cells lining bone were removed, and compared this to whole-bone samples, in which bone marrow was retained (Fig. 3a). The expression of genes encoding established osteocyte proteins were among the most enriched in osteocyte-enriched bone samples (Fig. 3b, Supplementary Data 2). Sost and Mepe were enriched by $>100$-fold and $D m p 1>40$ fold $^{38}$, whereas the expression of housekeeping genes was unaffected by cell composition and genes typically expressed in bone marrow cells were depleted in osteocyte-enriched samples (Fig. 3b).

Next, we fitted a four component Gaussian Mixture Model (GMM) to the distribution of gene enrichment and used this to calculate a threshold of osteocyte-enrichment. This model identified 1777 genes with significantly enriched expression in osteocytes (Fig. 3b and Supplementary Data 2). As an additional level of stringency 538 genes enriched in bone marrow or tissues that could contaminate the osteocyte enrichment strategy, such as blood or muscle (Fig. 3c and Supplementary Data 2) ${ }^{13}$, were excluded leaving 1239 genes significantly enriched for expression 

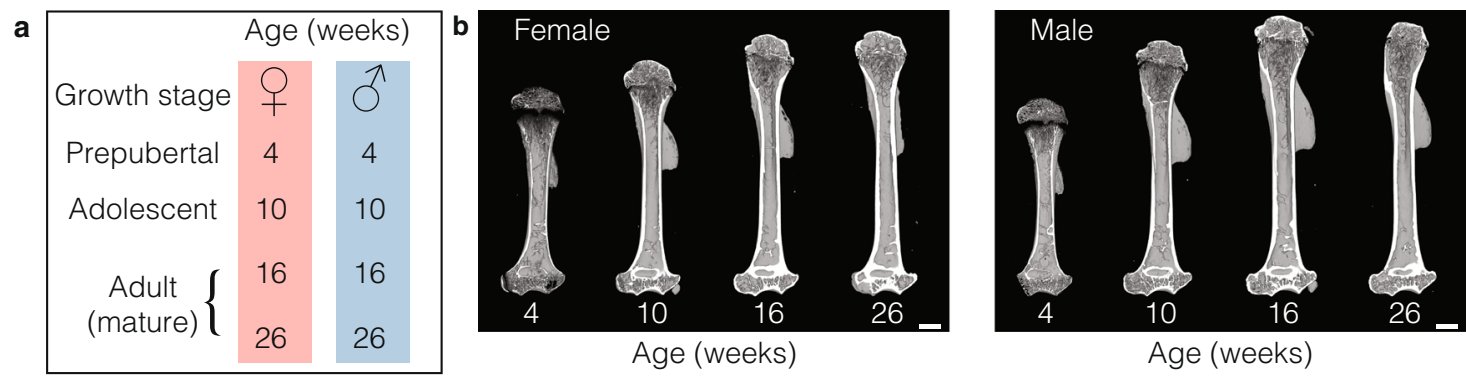

C

Female

Male

\begin{tabular}{|c|c|c|c|c|c|c|c|c|c|c|}
\hline $\begin{array}{l}\text { Gene } \\
\text { type }\end{array}$ & $\begin{array}{c}4 \text { weeks } \\
(\#)\end{array}$ & $\begin{array}{c}10 \text { weeks } \\
(\#)\end{array}$ & $\begin{array}{c}16 \text { weeks } \\
(\#)\end{array}$ & $\begin{array}{c}26 \text { weeks } \\
(\#)\end{array}$ & $\begin{array}{c}4 \text { weeks } \\
(\#)\end{array}$ & $\begin{array}{c}10 \text { weeks } 1 \\
(\#)\end{array}$ & $\begin{array}{c}16 \text { weeks } \\
(\#)\end{array}$ & $\begin{array}{l}26 \text { weeks } \\
(\#)\end{array}$ & $\begin{array}{l}\text { Any } \\
(\#)\end{array}$ & $\begin{array}{l}\text { All } \\
(\%)\end{array}$ \\
\hline Protein & 12370 & 12716 & 12874 & 12910 & 12353 & 12759 & 12912 & 12937 & 13592 & 86 \\
\hline IncRNA & 649 & 723 & 724 & 809 & 650 & 714 & 798 & 773 & 1150 & 36 \\
\hline TEC & 414 & 436 & 446 & 484 & 406 & 463 & 475 & 458 & 571 & 55 \\
\hline Novel & 57 & 56 & 49 & 66 & 52 & 52 & 71 & 57 & 125 & 18 \\
\hline Other & 37 & 45 & 44 & 44 & 41 & 39 & 50 & 38 & 60 & 47 \\
\hline Total & 13527 & 13976 & 14137 & 14313 & 13502 & 14027 & 14306 & 14263 & 15498 & 81 \\
\hline
\end{tabular}

d i

$\begin{array}{lllll}-100 & -50 & 0 & 50 & 100\end{array}$

ii

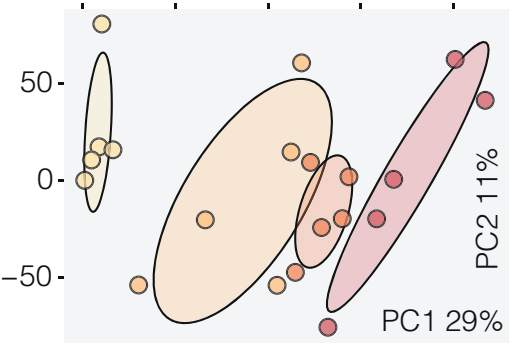

separation

ii $\quad-100 \quad-50 \quad 0 \quad 50 \quad 100$

$\frac{\text { Mean Dist }}{4-10 \quad 86}$

$10-16 \quad 44$

16-26 49

\begin{tabular}{|cccc|}
\hline Female \\
0 & 4 & 0 & 16 \\
0 & 10 & 0 & 26 \\
\hline
\end{tabular}
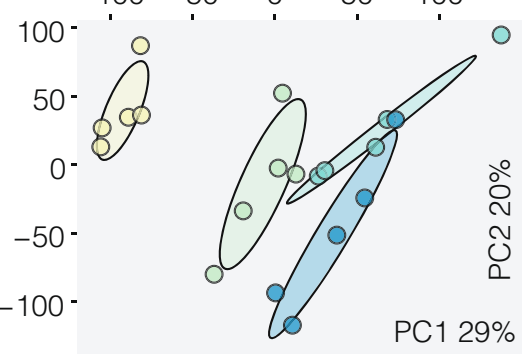

Separation

\begin{tabular}{c}
\hline Mean Dist \\
\hline $4-10 \quad 101$
\end{tabular}

10-16 82

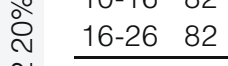

e i

ii -100

$0 \quad 100$ iii

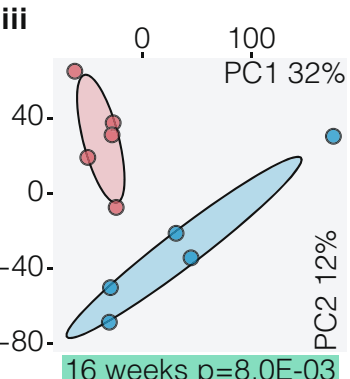

iv

$\begin{array}{lll}29 \% & 010026\end{array}$
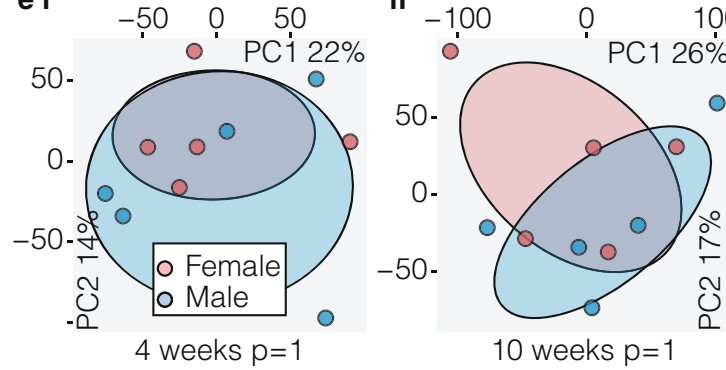

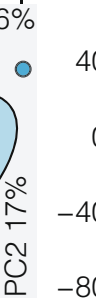

10 weeks $p=1$

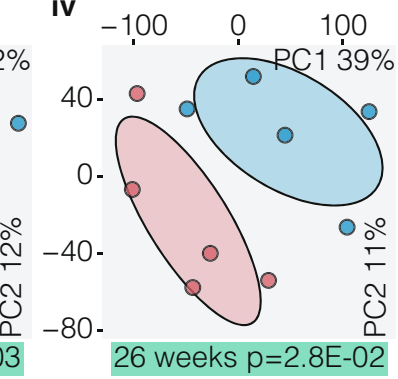

Fig. 2 The osteocyte transcriptome changes with sex and age. a Experimental design used to define the osteocyte transcriptome in each sex during skeletal maturation. $\mathbf{b}$ Representative micro-CT images of humeri from female and male mice at different ages. $\mathbf{c}$ The number of active genes and biotype composition of the osteocyte transcriptome at different ages in each sex. Any reflects the number of genes actively expressed in any ages or sexes, All reflects the percentage of genes active in any age or sex that are active in all ages and sexes. TEC $=$ to be experimentally confirmed. $\mathbf{d}$ Principal component (PC) analysis of samples clustered by age in female (i) and male (ii) mice. The percentage of total variance explained by individual PCs is shown. Dots represent individual biological replicates and ellipses represent $50 \%$ confidence intervals for each age. Dist $=$ Euclidean distance between cluster centroids (mean). $P$-values were calculated using Hotelling's $t$-test (one-sided, Bonferroni adjusted), clusters with significant separation $(P<0.05)$ between centroids are highlighted in green. e Principal component analysis of samples clustered from both sexes at 4 (i), 10 (ii), 16 (iii) and 26 (iv) weeks of age. The percentage of total variance explained by individual PCs is shown. Dots represent individual biological replicates and ellipses represent $50 \%$ confidence intervals for each sex. $P$-values were calculated using Hotelling's $t$-test (one-sided, Bonferroni adjusted), clusters with significant separation $(P<0.05)$ between centroids are highlighted in green.

in osteocytes (Fig. 3d and Supplementary Data 5). In total, $85 \%$ of these genes showed moderate to high expression specificity ${ }^{29}$, using the Tau measure, within the osteocyte network relative to 12 non-skeletal tissues (Fig. 3e). Furthermore, osteocyte-enriched genes were highly expressed in osteocytes relative to osteoblasts $\left(P=1.1 \times 10^{-38}\right)$ and bone-lining cells $\left(P=1.1 \times 10^{-43}\right)$ in a publicly available microarray dataset (Fig. 3f ${ }^{39}$. Using this pipeline we thus defined a list of 1239 genes whose expression is enriched in osteocytes relative to bone marrow and other cells in the osteoblast lineage. We defined this profile of genes as the osteocyte transcriptome signature (Supplementary Data 5 and Fig. 3d).

The majority of osteocyte transcriptome signature genes have no known function in the skeleton. Analysis of the osteocyte transcriptome signature showed it was enriched with genes associated with skeletal biological processes in the GO database $^{40}$ (4.5-fold-enrichment (FE), $\left.P=1.0 \times 10^{-67}\right)$, and 


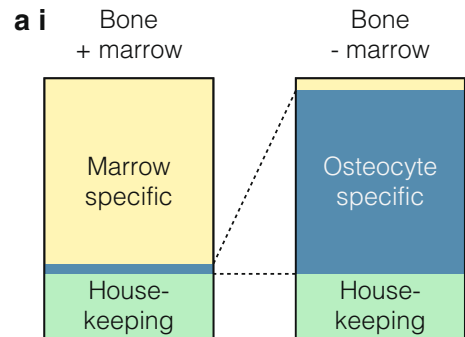

ii

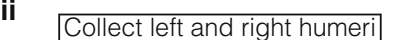
(wild-type mice $n=5$ )

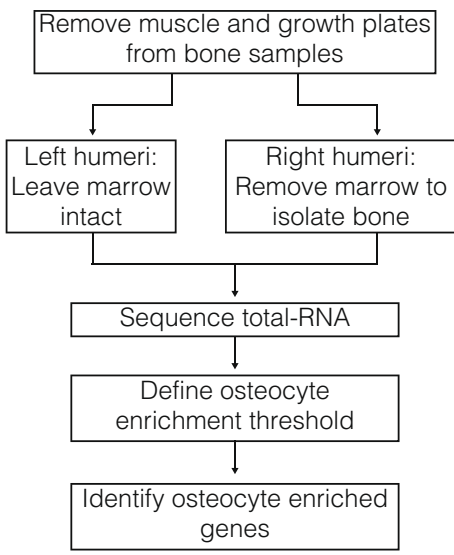

C
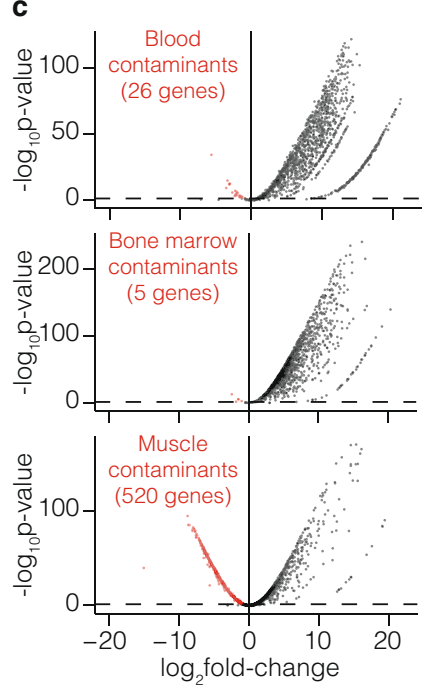

b i

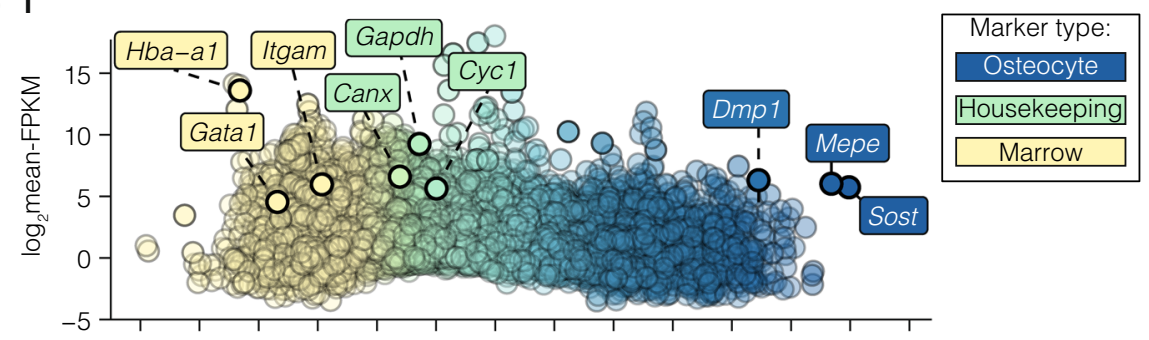

ii
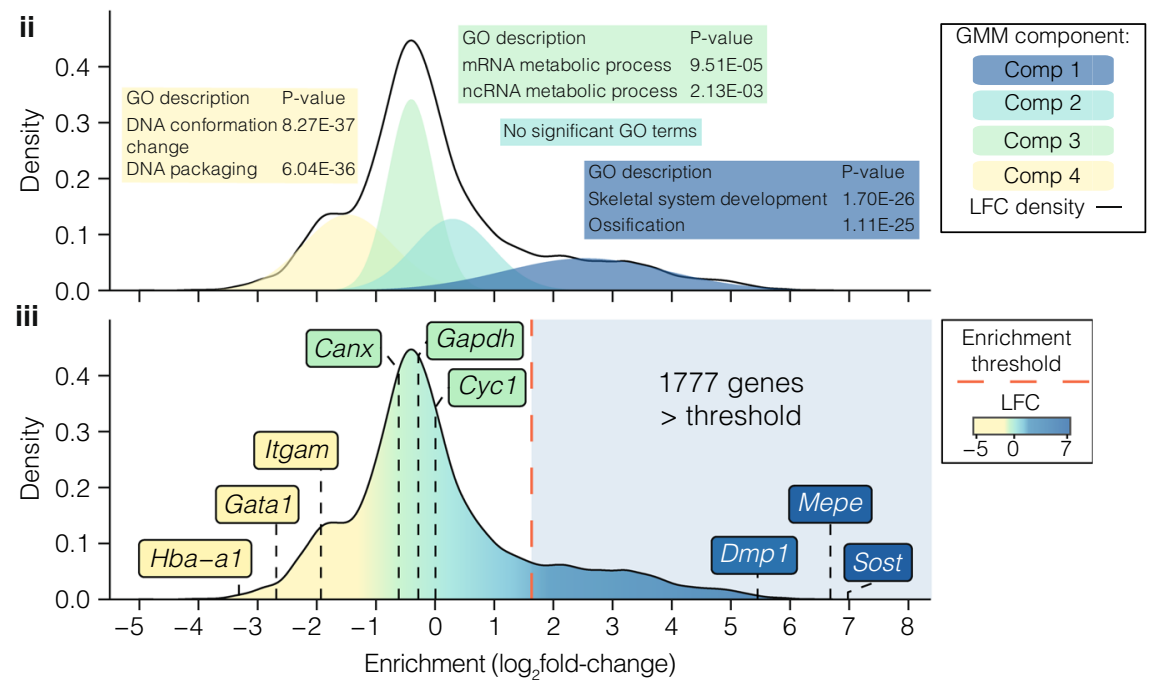

d

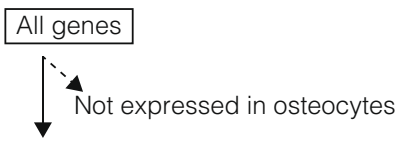

Genes expressed in osteocytes 16132 genes
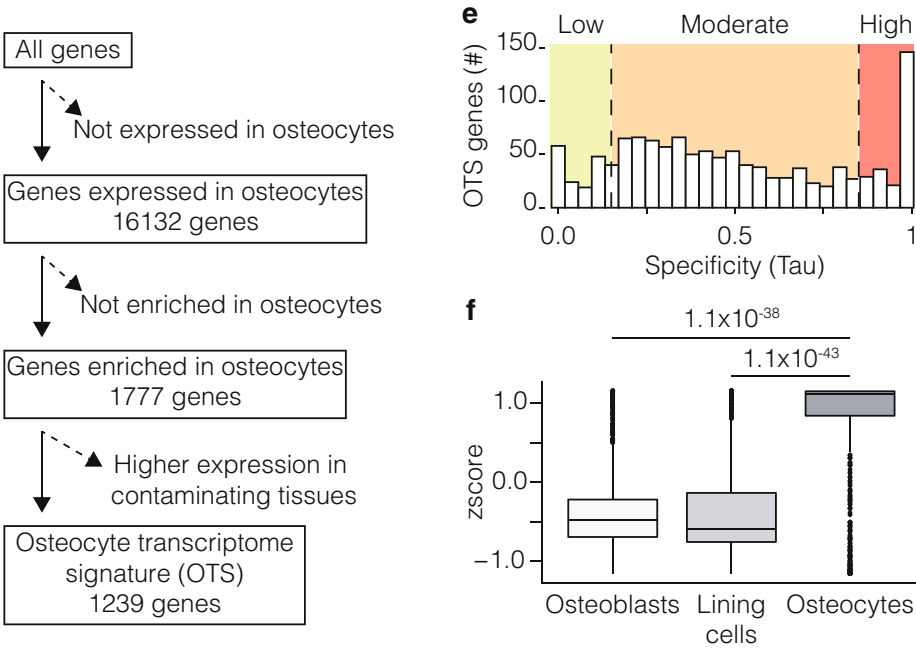

with skeletal phenotypes in the Mouse Genome Informatics database $^{41}$ (MGI, $2.7 \mathrm{FE}, P=4.7 \times 10^{-35}$ ). This included Sost, $D k k 1$, Mepe and Dmp1, genes known to be highly expressed in osteocytes, and genes with an established role in the skeleton, such as osteoprotegerin (Tnfsf11b), Wingless-type family member-1 (Wnt1 $)^{42}$, fibroblast growth factor-9 (Fgf9) ${ }^{43}$ and Iroquois homeobox protein 5 (Irx5) ${ }^{44}$ (Fig. 4a and Supplementary Fig 6). Interestingly, Tnfsf11 encoding RANKL (the ligand for receptor activator of $\mathrm{NFkB}$ ), was expressed by osteocytes but not present in the osteocyte transcriptome signature (Supplementary Fig 6). A limited number of genes were not annotated with skeletal terms in the GO database, but have been reported to have a role in the skeleton (denoted as 'reported') (Fig. 4a). They include the Wnt-regulator notum (Notum), which regulates bone formation ${ }^{45-47}$ and a distintegrin and metalloproteinase like member (Adamtsl2), which is implicated in geleophysic dysplasia 1 (OMIM 231050) ${ }^{48}$. The majority of osteocyte transcriptome signature genes $(78 \%, n=$ 968) had not previously been shown to have a role in the skeleton ('unannotated' in Fig. 4a).

In addition to known genes, forty-nine novel genes were actively expressed in osteocytes (Supplementary Fig 7a and Supplementary Data 6). Eleven were present in the osteocyte transcriptome signature, (Supplementary Fig 7b), including 7 that were absent from 12 other tissues (Supplementary Fig 7c). The multiple exons and splicing patterns suggested posttranscriptional processing of transcripts, while analysis of sequence-coding potential indicated they were all non-coding genes (Supplementary Fig 7c). Thus, the osteocyte transcriptome signature expands the repertoire of genes whose expression is enriched in osteocytes and includes known and novel genes. 
Fig. 3 Defining an osteocyte transcriptome signature. a Experimental design used to identify genes with enriched expression in osteocytes. Diagram illustrating the strategy used to identify genes enriched for expression in osteocytes in bone samples enriched with osteocytes relative to intact bone samples in which bone marrow and cells lining bone were retained (i). Experimental workflow used to identify genes enriched for expression in osteocytes (ii). b Gene-enrichment in osteocyte-enriched bone samples relative to whole-bone samples distinguished genes known to be expressed in osteocytes (blue) from housekeeping genes (green) and genes expressed in marrow cell populations (yellow) (i). Enrichment for each individual gene is shown as a dot and plotted as a function of normalised gene expression ( $\log _{2}-\mathrm{FPKM}$ ). The four component gaussian mixture model fitted to the density distribution of gene-enrichment in (i) used to define the osteocyte enrichment threshold (ii). Each component is denoted by a separate colour. The top two Gene Ontology biological processes associated with genes in each component are illustrated in coloured boxes with $p$-values (Bonferroni adjusted) calculated by hypergeometric test. (ii). 1777 genes above the osteocyte-enrichment threshold (indicated by the red dashed line) were significantly enriched for expression in osteocytes (iii). LFC $=\log _{2}$ fold-change. $\mathbf{c}$ Volcano plots comparing the expression of osteocyte-enriched genes (from $\mathbf{b}$ ) with blood (i), bone marrow (ii) and muscle (iii) ${ }^{13}$. Dashed lines represent $p<0.05$ cutoff. Genes significantly enriched in these tissues relative to osteocytes are identified as red dots. $P$-values were calculated in the original publishing article ${ }^{13}$ by F-test with Benjamini-Hochberg adjustment. $\mathbf{d}$ Filtering pipeline used to define the 1239-gene osteocyte transcriptome signature (OTS). The number of genes remaining at each filtering stage is indicated. e Expression specificity ${ }^{29}$ (Tau) of OTS genes relative to other organs and tissues ${ }^{28}$. Genes with Tau $<0.15=$ low expression specificity in osteocytes $($ green), $0.15 \leq$ Tau $\leq 0.85=$ moderate expression specificity (orange), while Tau $>0.85=$ high expression specificity (red). $\mathbf{f}$ OTS genes were enriched for expression in osteocytes relative to osteoblasts and bone-lining cells isolated by laser-capture microdissection. Tukey boxplots show a summary of median OTS gene expression values in each cell type calculated across $n=3$ biological replicates from a single experiment. Boxes indicate median and interquartile range (IQR) of scaled, normalised gene expression values, whiskers denote values $\pm 1.5 \times I Q R$ and outlier values beyond this range are shown as individual points. $P$-values (two-tailed, Benjamini and Hochberg adjusted) were calculated by competitive gene set test accounting for inter-gene correlation (CAMERA) ${ }^{105}$.

Analysis of GO terms enriched in the osteocyte transcriptome signature identified eight semantically similar clusters of related processes (Fig. 4b and Supplementary Data 7). Cluster 1 was enriched with processes associated with ossification (GO:0001503, $\left.P=2.3 \times 10^{-41}\right)$, cluster 2 extracellular matrix organisation (GO:0030196, $P=2.8 \times 10^{-41}$ ), cluster 3 skeletal system development (GO:0001501, $P=2.9 \times 10^{-38}$ ), cluster 4 osteoblast differentiation (GO:0001649, $P=4.2 \times 10^{-22}$ ) and cluster 6 mesenchymal cell proliferation (GO:0010463, $P=3.44 \times 10^{-15}$ ), whereas, clusters 7 and 8 were enriched with processes associated with signalling, including wnt signalling pathway (GO:0016055, $P=3.0 \times 10^{-13}$ ) and cellular response to BMP stimulus (GO:0071773, $P=2.0 \times 10^{-10}$, Fig. 4 b). GO term analysis also identified cluster 5 which was enriched with terms associated with axon guidance including axon development (GO:0061564, $P=$ $9.6 \times 10^{-22}$ ) and axonogenesis (GO:0007409, $P=1.0 \times 10^{-20}$; Fig. 4b). Axon guidance (mmu04360, $P=7.8 \times 10^{-17}$ ) was also the top-ranked KEGG pathway (Supplementary Fig 8a). This included genes in the Semaphorin, Ephrin, Netrin and Slit signalling families and their respective receptors Plexins, Ephreceptors, Uncoordinated-5 (Unc-5) and Roundabout (Robo), which are pivotal regulators of axonal guidance (Supplementary Fig $8 b$ ). Since axon guidance directs the formation of the intercellular neuronal network ${ }^{49}$, we hypothesised that this pathway is a key molecular programme required for osteocyte network formation.

To examine this, we investigated temporal patterns of osteocyte transcriptome signature gene expression in the in vitro IDG-SW3 model of osteocyte cell differentiation in which cells differentiate from late osteoblast-like cells, via early osteocytes, to late osteocytic cells ${ }^{50}$. Three clusters of genes were identified (Fig. 4c and Supplementary Data 5), an early expression cluster - 377 genes most highly expressed in osteoblast-like cells, but down-regulated as cells transition to early and mature osteocytes (Fig. 4c, d); an early activation cluster - 453 genes up-regulated in early osteocytes, and which remained expressed in mature osteocytes (Fig. 4c, d); and a maturation cluster - 409 genes up-regulated in mature osteocytes (Fig. 4c, d). The early expression cluster was enriched for processes associated with extracellular matrix organisation (GO:0030198, $P=7.51 \times 10^{-16}$, Fig. 4e) and the maturation cluster with ossification (GO:0001503, $P=$ $3.40 \times 10^{-26}$, Fig. 4 e). The early activation cluster was associated with axon development (GO:0061564, $P=1.31 \times 10^{-13}$ ), axonogenesis (GO:0007409, $P=1.38 \times 10^{-13}$ ) and axon guidance (GO:0007411, $P=2.43 \times 10^{-9}$, Fig. $4 \mathrm{e}$ ). The upregulation of these processes coinciding with early osteocyte differentiation and sustained expression in late osteocytes suggests axonal guidance pathways are important in the formation and maintenance of the osteocyte network.

Osteocyte transcriptome signature genes control bone structure and function. To establish whether osteocyte transcriptome signature genes have a functional role in the skeleton, we screened mice with single gene deletions that have undergone detailed skeletal phenotyping by the Origin of Bone and Cartilage Disease (OBCD) programme (http://www.boneandcartilage. com/bonepipeline.html). Structural and functional bone phenotyping has been performed in 733 knockout mouse lines, of which 64 had deletions of genes present in the osteocyte transcriptome signature (Fig. 5a and Supplementary Data 8). In total, $26(41 \%)$ of these knockout lines had either structural and/or functional skeletal phenotypes. Eleven were in genes with established roles in the skeleton, including Daam $2^{25}$ and $P l s 3^{51}$. Fifteen were in genes not annotated with skeletal terms or phenotypes in the GO or MGI databases, suggesting they are novel regulators of bone structure and/or function (Fig. 5a, Supplementary Data 8). These included the activator of transcription and development regulator (Auts2), a regulator of neuron migration $^{52}$, dapper antagonist of beta-catenin 3 (Dact3), which controls Wnt signalling 53,54 and low-density lipoprotein receptor class A domain containing protein (Ldlrad4), an inhibitor of TGF-Beta signalling 55 (Fig. 5a). Genes in the MGI database, but without functional evidence of a role in the skeleton such as cortactin binding protein $2(C t t n b p 2)$ (Fig. 5a), a regulator of dendrite arbourisation ${ }^{56}$, were also identified. Auts $2^{+/}$mice had decreased femoral bone length and cortical diameter (Fig. 5b, c), whereas Cttnbp2 $2^{-/-}$had normal femoral length, BMC and functional parameters but increased vertebral BMC and maximum load (Fig. 5b,c,e). Dact $3^{-1-}$ had normal femoral bone length, BMC, bone structural parameters and functional parameters, however, BMC was increased in the vertebra and this was accompanied by increased yield load (Fig. 5b, c, e). Ldlrad4 $4^{-1-}$ had normal bone length, BMC and functional parameters but had decreased bone volume (BV/TV), decreased trabecular number (Tb.N) and increased trabecular separation (Tb.Sp; Fig. 5b, c). High resolution micro-CT analysis of osteocyte lacunae showed that deletion of Cttnbp2 and Ldlrad4 caused an 
a

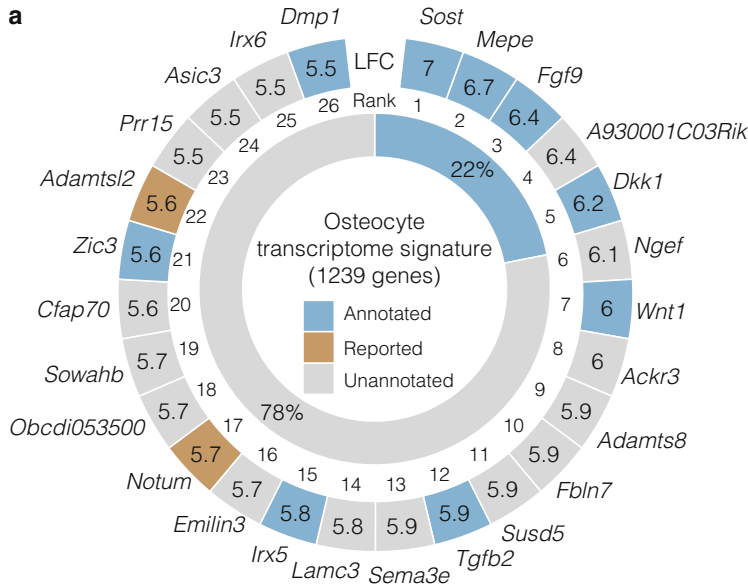

b

GO:0001503: ossification

GO:0030278: regulation of ossification GO:0030198: extracellular matrix organization GO:0043062: extracellular structure organization GO:0001501: skeletal system development GO:0061448: connective tissue development GO:0001649: osteoblast differentiation

GO:0051961: -ve regulation of nervous system development GO:0061564: axon development GO:0007409: axonogenesis GO:0010463: mesenchymal cell proliferation GO:0001667: ameboidal-type cell migration GO:0016055: Wnt signaling pathway GO:1905114: surface receptor involved in cell-cell signaling* GO:0071773: cellular response to BMP stimulus GO:0071772: response to BMP

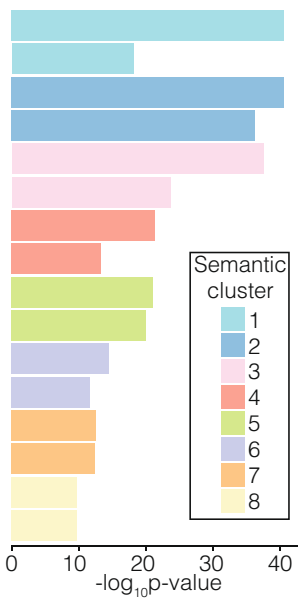

c

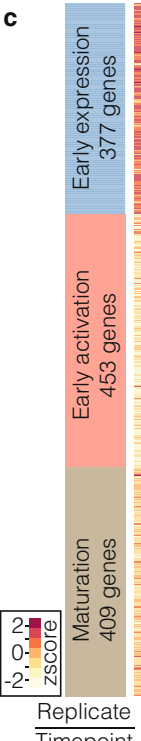

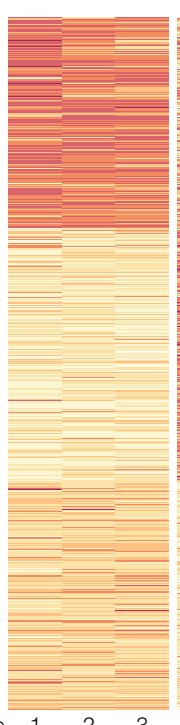

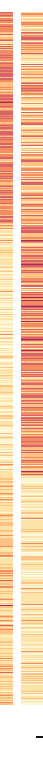

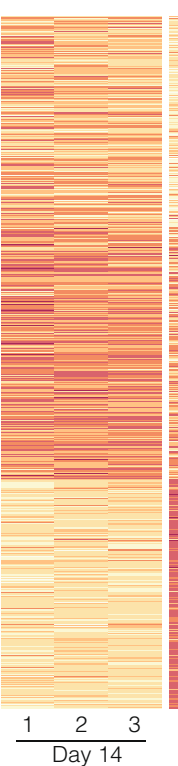
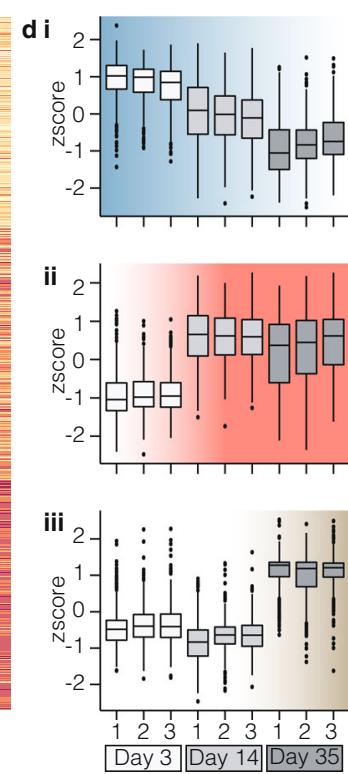

e i Early Expression GO Biological Processes

GO:0030198 extracellular matrix organization GO:0043062 extracellular structure organization GO:0001525 angiogenesis GO:0051216 cartilage development GO:0061448 connective tissue development

\begin{tabular}{ll} 
ii & \\
\hline Early Activation GO Biological Processes & $1.31 \mathrm{E}-13$ \\
GO:0061564 axon development & $1.38 \mathrm{E}-13$ \\
GO:0007409 axonogenesis & $2.43 \mathrm{E}-09$ \\
GO:0007411 axon guidance & $2.74 \mathrm{E}-09$ \\
GO:0050770 reuron projection guidance & $8.39 \mathrm{E}-09$ \\
\hline
\end{tabular}

\begin{tabular}{ll}
${ }$ Maturation GO Biological Processes $}$ & P-value \\
\hline GO:0001503 ossification & $3.40 \mathrm{E}-26$ \\
GO:0001501 skeletal system development & $2.39 \mathrm{E}-20$ \\
GO:0001649 osteoblast differentiation & $5.79 \mathrm{E}-17$ \\
GO:0060348 bone development & $6.27 \mathrm{E}-17$ \\
GO:0030278 regulation of ossification & $8.82 \mathrm{E}-15$ \\
\hline
\end{tabular}

Fig. 4 Genes and control pathways identified by the osteocyte transcriptome signature. a The top 26 osteocyte transcriptome signature genes most enriched in osteocytes (outer ring). Genes annotated with either a skeletal biological process (GO database) or skeletal phenotype (MGI database) are highlighted blue; genes reported with experimental evidence of a role in the skeleton are brown, whereas unannotated genes without a skeletal annotation

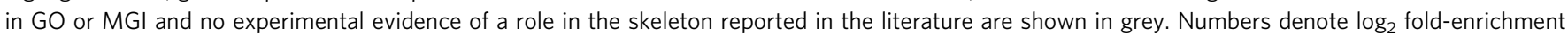
(LFC) in gene expression in osteocytes. The proportion of all osteocyte transcriptome signature genes annotated (blue) or unannotated (grey) with a skeletal annotation in $\mathrm{GO}$ or $\mathrm{MGI}$ is shown in the inner ring. $\mathbf{b}$ Clusters $(n=8)$ of semantically similar biological processes (GO) significantly enriched in the osteocyte transcriptome signature are identified by different colours (Bonferroni-corrected $P<0.05$ ). The top 2 terms (by $P$-value) in each of the eight clusters are listed. c Heatmap showing clustering of osteocyte transcriptome signature genes based on distinct co-expression patterns during osteocyticdifferentiation ${ }^{50}$ of the IDG-SW3 cell-line, from osteoblast-like cells (day 3) to early (day 14) and mature osteocytes (day 35). The name and number of genes for each cluster are labelled. Cluster colours correspond to subsequent panels (d-e). d Changes in early expression cluster (i), early activation cluster (ii) and maturation cluster (iii) of genes during osteocyte differentiation. Tukey boxplots show the distribution of gene expression values for each cluster in cells from three independent experiments $(n=3)$. Boxes indicate median and interquartile range (IQR) of scaled, normalised gene expression values, whiskers denote values $\pm 1.5 \times \mathrm{QR}$ and outlier values beyond this range are shown as individual points. e Biological processes (BP) enriched in the early expression cluster (i), the early activation cluster (ii) and the maturation cluster (iii). The top 5 GO BP terms in each cluster with P-values (Bonferroni adjusted) calculated by hypergeometric test.

increase in lacuna volume with increased numbers of large lacunae and fewer smaller lacunae, deletion of Auts 2 enhanced lacuna sphericity, whereas Dact3 deletion had no effect on size distribution or sphericity (Fig. 5d and Supplementary Fig 9). Auts2 and Ldlrad4 expression was higher in osteocyte-enriched bone than in other tissues. By contrast, expression of Cttnbp2 and Dact 3 was highest in brain and/or adrenal gland, respectively (Supplementary Fig 10), suggesting that deletion of these two genes might result in both direct local effects on the skeleton and secondary additional contributions via their expression in other tissues.
In addition to annotated genes, mice with targeted deletion of the novel, non-annotated genes Obcdi008175 and Obcdi042809 also had abnormal skeletal phenotypes, whereas, Obcdi007392 deficient mice had normal bones (Supplementary Fig 11). Male Obcdi008175 ${ }^{-1-}$ mice had increased femoral BMC and female Obcdi008175-1- mice had decreased femoral length and vertebral strength. By contrast, female Obcdi042809-I- mice had decreased vertebral BMC and strength (Supplementary Fig 11).

These data demonstrate that the osteocyte transcriptome signature identifies genes not previously known to affect bone, 
a
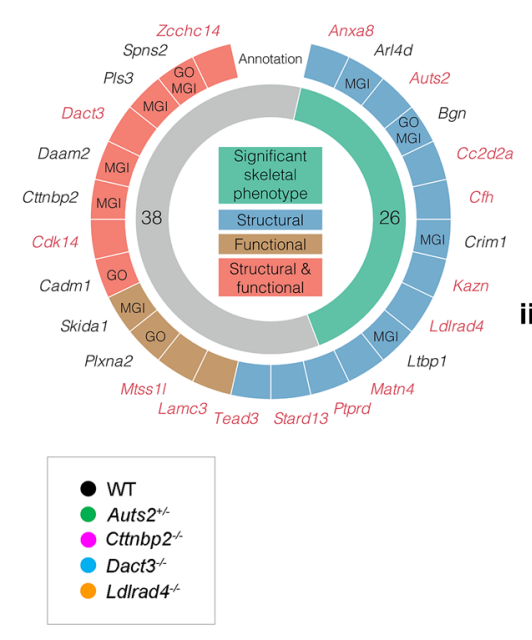

c i
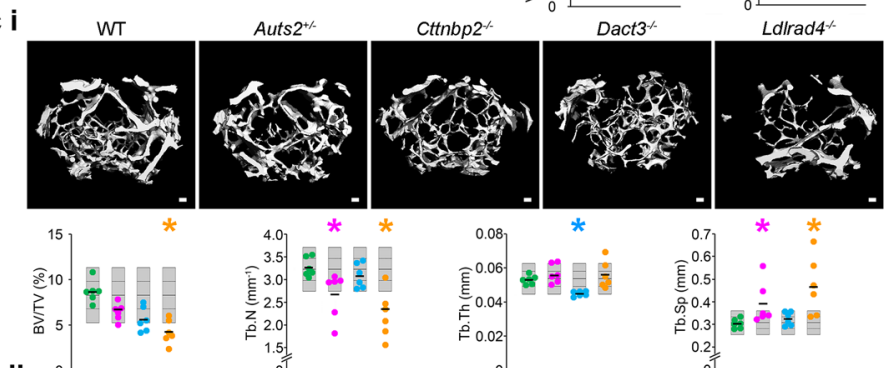

ii

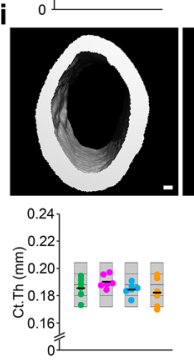

d
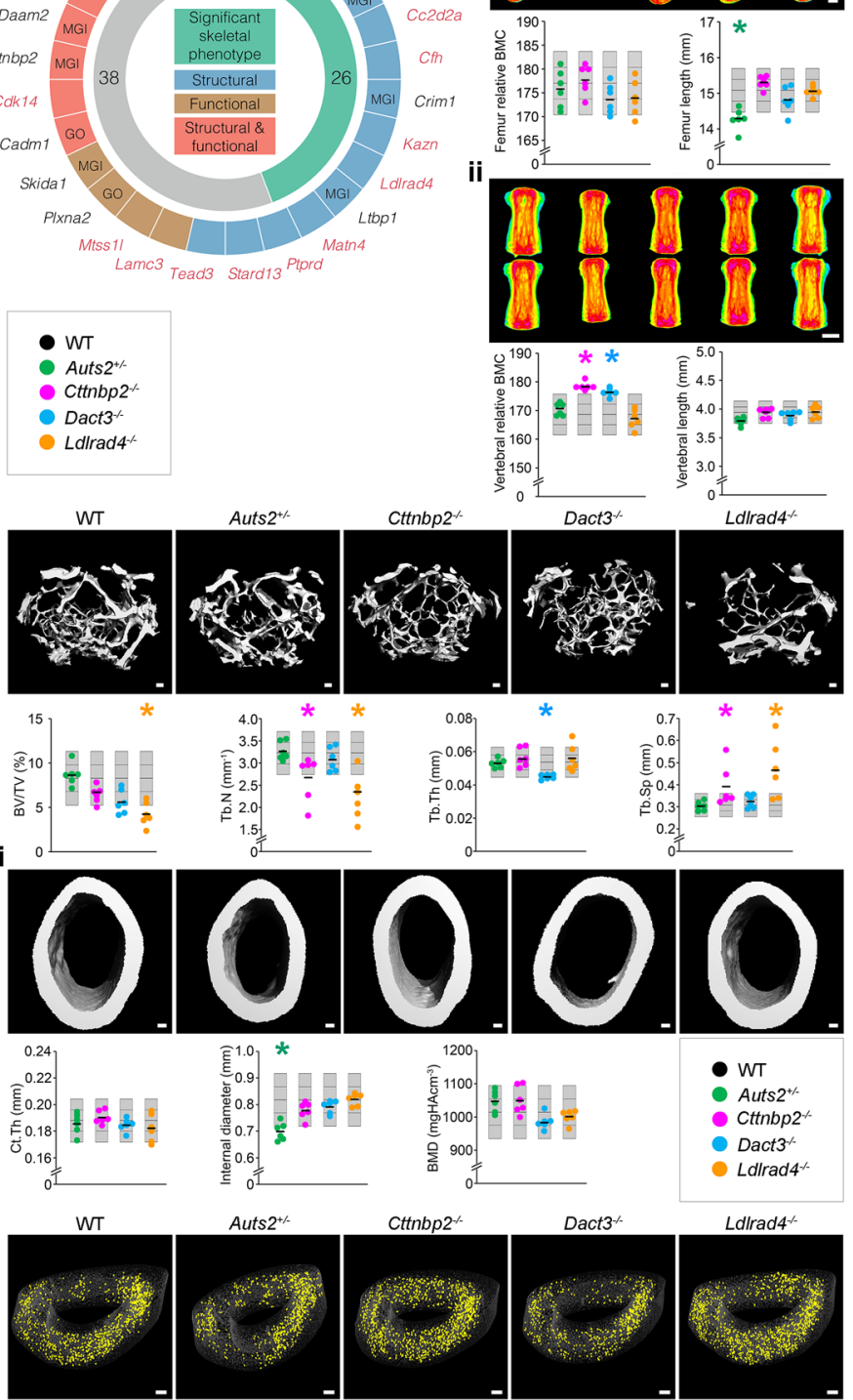

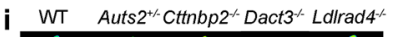
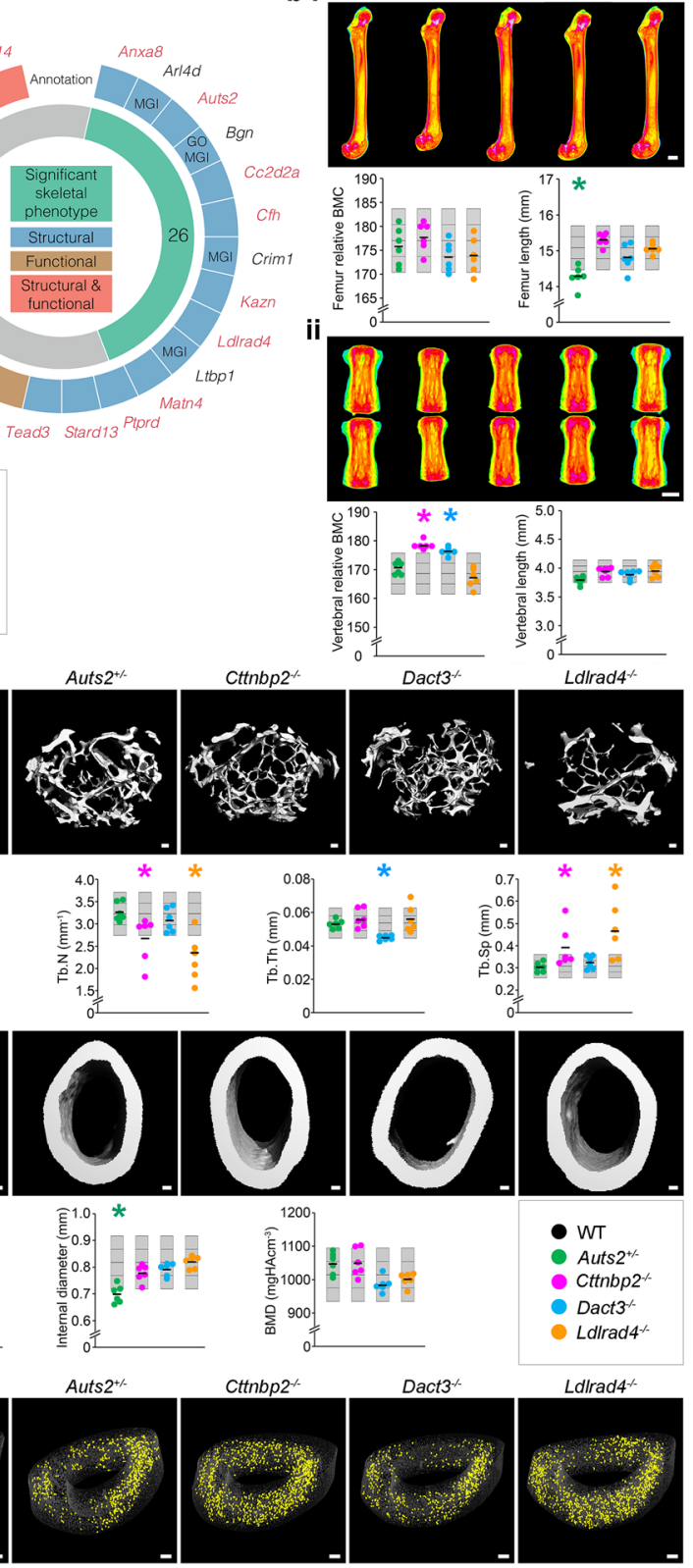

Ldirad4-
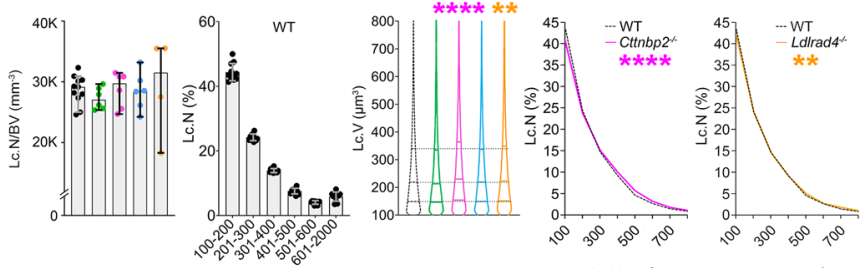

e
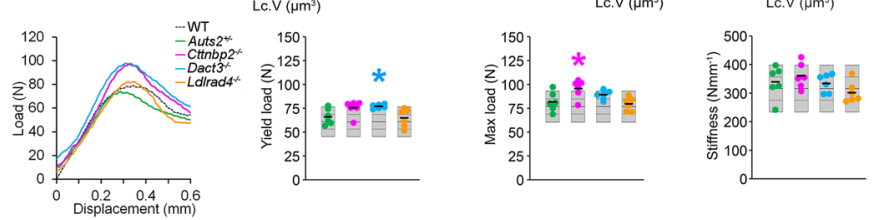

including novel genes, that play important structural and functional roles in the skeleton.

Osteocyte transcriptome signature genes are associated with rare skeletal disorders. Given their functional role in mice, we next hypothesised that the osteocyte transcriptome signature would be enriched for genes that cause rare monogenic skeletal disorders in humans. Three-hundred and ninety two of 432 skeletal dysplasia-causing gene-orthologs ${ }^{21}$ ( $\left.\sim 91 \%\right)$ were actively expressed in osteocytes supporting a key role for osteocytes in skeletal disease. Mutations in 90 genes present in 
Fig. 5 Deletion of osteocyte transcriptome signature genes affects bone structure and function. a The 26 osteocyte transcriptome signature genes with outlier skeletal phenotypes in the Origins of Bone and Cartilage Disease (OBCD) database of single gene deletions in mice (outer ring). Deletion of genes in blue affected bone structure, genes in brown affected bone function and genes in red both structure and function. Those annotated in either GO or MGI are indicated. Genes in red have a significant outlier phenotype when deleted in knockout mice but are not annotated with skeletal function or phenotype in either GO or MGI databases. The inner ring denotes the number of genes with (green) and without (grey) an outlier skeletal phenotype when deleted in mice in the OBCD database. $\mathbf{b}$ Representative quantitative X-ray microradiographic images from the femurs (i) and vertebrae (ii) of adult, female wild-type (WT), Auts2 $2^{+/-}$, Cttnbp2 $^{-/-}$, Dact3 ${ }^{-/-}$and Ldlrad4 $4^{-/-}$mice $(n=6$ mice per genotype). Scale bar $=1 \mathrm{~mm}$. Dot plots illustrate individual parameters.

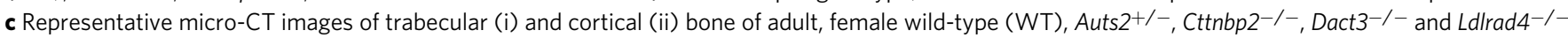
mice ( $n=6$ mice per genotype). Scale bar $=100 \mu \mathrm{m}$. Dot plots illustrate bone volume as a proportion of tissue volume (BV/TV), trabecular number (Tb. $\mathrm{N}$ ), trabecular thickness (Tb.Th), trabecular separation (Tb.Sp), cortical thickness (Ct.Th), internal endosteal diameter and bone mineral density (BMD). d Representative micro-CT images showing large osteocyte lacunae $\left(401-800 \mu \mathrm{m}^{3}\right)$ in tibia cortical bone from adult, female WT $(n=11)$, Auts $2^{+/}-(n=$ 6), Cttnbp2 ${ }^{-/}(n=6)$, Dact3 $^{-/-}(n=6)$ and Ldlrad4 ${ }^{-/}(n=4)$ mice. Scale bar $=100 \mu \mathrm{m}$. Graphs show osteocyte lacunae number per bone volume (Lc. N/BV) and distribution of lacunae volumes (LC.V) in WT mice (median and 95\% confidence intervals). Violin plot shows distribution of Lc.V in the four knockout mouse lines compared to WT and graphs show relative frequency distribution of Lc.V in Cttnbp2 $2^{-1-}$ and Ldlrad4 $4^{-/-}$mice compared to WT.

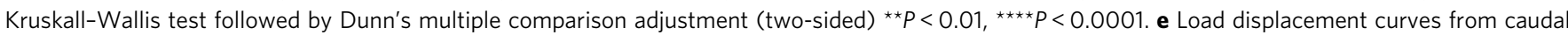
vertebrae compression testing in adult female WT, Auts2 $2^{+/}$, Cttnbp2 ${ }^{-/-}$, Dact $3^{-/-}$and Ldlrad4 $^{-/-}$mice. Dot plots show yield load, maximum load and stiffness. For each variable in $\mathbf{b}, \mathbf{c}$ and $\mathbf{e}$ the mean (solid centre lines), \pm 1.0 SD (dotted lines) and \pm 2.0 SD. (grey boxes) for WT mice ( $n=320$ ) are shown. Individual data-points for each parameter in Auts2 ${ }^{+/-}$, Cttnbp2 ${ }^{-/-}$, Dact3 ${ }^{-/-}$and Ldlrad4 ${ }^{-/-}$lines are shown as green, pink, blue and orange dots, respectively ( $n=6$ biologically independent mice per genotype). The mean value for each individual phenotype parameter is indicated by a solid bold line. Mean parameters that lie greater or less than two standard deviations from the WT reference mean are considered outliers and are indicated by an asterisk $\left.{ }^{\star}\right)$, coloured according to the individual mouse line.

the osteocyte transcriptome signature cause 168 of the 612 skeletal disorders annotated in the nosology of genetic skeletal disorders ${ }^{21}$ (3-FE, $P=2.4 \times 10^{-22}$, Fig. 6a and Supplementary Data 9). Nevertheless, osteocyte transcriptome signature genes were not uniformly involved in all of the skeletal disease groups (Fig. 6b). For example, the osteogenesis imperfecta and decreased bone density group was one of the groups most enriched with signature genes (18/33 casual genes in the signature, $\left.P=7 \times 10^{-13}\right)$. Indeed, all 19 genes known to cause osteogenesis imperfecta (OI $)^{57-59}$ were actively expressed in osteocytes and 14 were present in the osteocyte transcriptome signature (Fig. 6c). Analysis of the temporal expression pattern of the 19 OI-genes in the IDG-SW3 in vitro cell differentiation dataset showed that 16 (84\%) were more highly expressed in late osteocytes relative to earlier stages in differentiation, including 13 osteocyte transcriptome signature genes (Supplementary Fig 12a). OI-genes were also more highly expressed in osteocytes isolated by laser-capture microdissection compared to bone-lining cells and osteoblasts, as well as other tissues (Supplementary Fig 12b, c). Furthermore, 13 of the 14 OI-genes present in the osteocyte transcriptome signature were up-regulated in osteocytes during skeletal growth but down-regulated at skeletal maturity, suggesting that these genes may have a role in osteocytes during bone development (Supplementary Fig 12d). Together these data demonstrate that the osteocyte transcriptome signature is highly enriched for genes that cause rare monogenic skeletal disorders. Thus, interrogation of the osteocyte transcriptome signature may inform candidate gene prioritisation in the study of families with rare bone disease in which the genetic basis is unknown.

The osteocyte transcriptome signature is enriched for human orthologs associated with skeletal disease susceptibility. Finally, we hypothesised that human orthologs of osteocyte transcriptome signature genes, identified in mice, are also enriched for genes associated with susceptibility to common skeletal diseases, such as osteoporosis and OA. To test this, the relationship between genetic variations surrounding human orthologs of osteocyte transcriptome signature genes and quantitative ultrasoundderived heel bone mineral density (eBMD) in a sample of 362,924 individuals from the UK Biobank (UKBB) $\operatorname{cohort}^{25}$ was examined using two methods: stratified linkage disequilibrium score regression (LDSC-SEG) ${ }^{60,61}$ and competitive gene set enrichment analysis ${ }^{62}$. LDSC-SEG analysis provided robust evidence of enrichment, demonstrating that genomic regions surrounding human osteocyte transcriptome signature gene orthologs contribute disproportionately to the SNP heritability of eBMD. The osteocyte transcriptome signature gene annotation $( \pm 20 \mathrm{~kb})$ spanned $5.1 \%$ of the genome (i.e. $65,332 / 1,142,435$ SNPs) and explained $\sim 12.5 \%\left(\mathrm{SE}=0.012, P=1.6 \times 10^{-7}\right)$ of the total estimated SNP heritability of eBMD (estimated previously ${ }^{25}$ at $\left.h^{2}{ }_{\mathrm{SNP}}=0.36, \mathrm{SE}=0.017\right)$. This corresponded to a 2.2 -fold $\left(\mathrm{SE}=0.21, P=1.6 \times 10^{-7}\right.$ ) enrichment for per-SNP heritability of eBMD.

Competitive gene-set analysis also detected strong enrichment and showed that, on average, human orthologs of osteocyte transcriptome signature genes exhibit stronger associations with eBMD than non-osteocyte transcriptome signature genes $(P=$ $\left.1.8 \times 10^{-13}\right)$. Enrichment was largely attributable to significant gene-level associations $\left(P<2.6 \times 10^{-6}\right)$ of $259 / 992$ osteocyte transcriptome signature orthologs $(26 \%)$ with eBMD (Fig. 7a and Supplementary Data 10). Mutations in 36 of these genes, with gene-level associations, cause monogenic skeletal dysplasia in humans (5.1-fold enrichment, $P=5.1 \times 10^{-16}$; Supplementary Data 10). We observed robust associations between SOST, DMP1 and MEPE $\left(P_{\mathrm{JOINT}}<2 \times 10^{-129}, P_{\mathrm{JOINT}}=2 \times 10^{-27}\right.$ and $P_{\text {JOINT }}=2 \times 10^{-86}$, respectively), genes enriched for expression in osteocytes. Given that these genes were reported as the closest gene to a lead variant in previous GWAS of $\mathrm{eBMD}^{25}$, we next investigated whether osteocyte transcriptome signature genes occurred nearest to lead variants more often than expected by chance. 110 osteocyte transcriptome signature orthologs were the closest genes to conditionally independent lead eBMD variants (2.8-fold enrichment, $P=2.4 \times 10^{-23}$ ) (Fig. 7a and Supplementary Data 10). These included 9 of the top 26 genes most enriched for expression in the osteocyte transcriptome signature (SOST, $M E P E, N G E F$, WNT1, ACKR3, TGFB2, SEMA3E, IRX5 and $D M P 1)$. Finally, we identified AUTS2 $\left(P_{\text {JOINT }}=6 \times 10^{-9}\right)$ as an osteocyte transcriptome signature gene that has not previously been implicated by GWAS (i.e. not within $1 \mathrm{Mb}$ of a lead eBMD variant), yet reached gene-wide significance in gene-level analysis and caused structural and/or functional skeletal abnormalities when deleted in mice (Fig. 5a-e). LDLRAD4 and CTTNBP2 $\left(P_{\text {JOINT }}=2 \times 10^{-20}\right.$ and $P_{\text {JOINT }}=2 \times 10^{-87}$ respectively $)$, which 


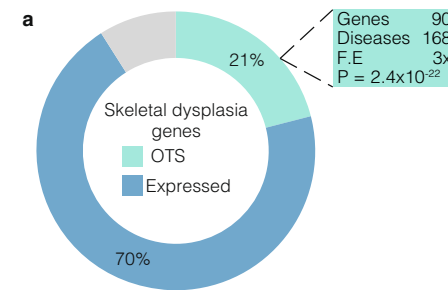

b

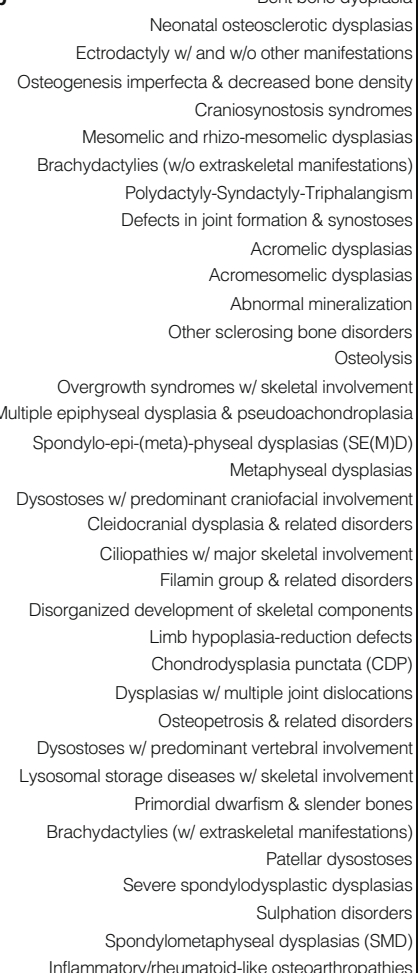

Inflammatory/rheumatoid-like osteoarthropathies

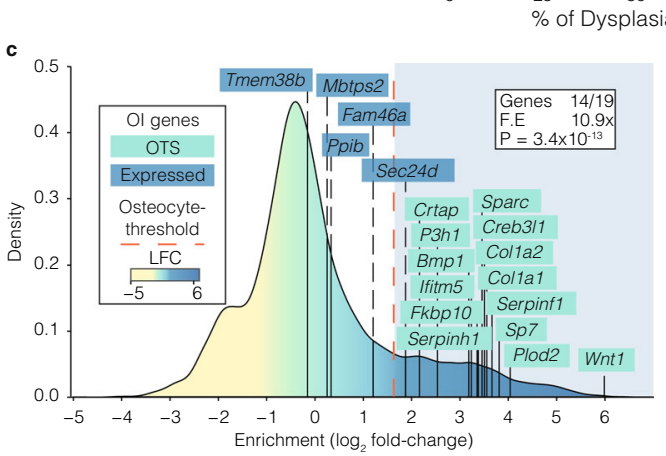

occur within $1 \mathrm{Mb}$ of previously identified eBMD loci, were also shown to result in an abnormal skeletal phenotype when deleted in mice. Together, this demonstrates that variants in osteocyte transcriptome signature gene-orthologs account for a significant proportion of the genetic variance that regulates $\mathrm{eBMD}$ and can help identify genes that affect skeletal structure and function.

Since changes in subchondral bone structure and mineralisation are pathognomonic in the development of $\mathrm{OA}^{63}$ we also sought to determine whether variants in osteocyte transcriptome signature orthologs were associated with $\mathrm{OA}$ in humans. A similar analytical approach was applied to 77,052 individuals with OA and 378,169 control subjects drawn from the UKBB and arcOGEN resources ${ }^{64}$. LDSC-SEG analysis ${ }^{60,61}$ showed that loci associated with human osteocyte transcriptome signature gene orthologs contribute to the heritability of $\mathrm{OA}$ at any site $\left(P=1.6 \times 10^{-7}\right)$, at the knee
Fig. 6 The osteocyte transcriptome signature is enriched with genes associated with rare genetic skeletal disorders. a Proportion of genes (\%) known to cause genetic skeletal disorders ${ }^{21}$ that have human orthologs either actively expressed in osteocytes (blue), present in the osteocyte transcriptome signature (OTS) (green) or not active in osteocytes (grey). $P$-value and fold-enrichment (FE) of osteocyte transcriptome signature genes among disease-causing genes are calculated under the hypergeometric distribution. b Proportion of genes (\%) in individual skeletal disease groups that have orthologs actively expressed in osteocytes (blue bars) or present in the osteocyte transcriptome signature (circles). Group size $=$ number of genes in each disease group. Groups with genes significantly enriched in the osteocyte transcriptome signature are coloured according to $P$-value (Bonferroni adjusted), calculated by hypergeometric test. Only groups with $\geq 3$ genes are represented. N.S. not significant. c Osteocyte-enrichment of genes associated with osteogenesis imperfecta (OI). Genes are coloured according to their identification as being actively expressed in osteocytes (blue) or present in the osteocyte transcriptome signature (green). Bonferroni-corrected $P$-value and foldenrichment (FE) of osteocyte transcriptome signature genes among Olcausing genes are calculated under the hypergeometric distribution.

$\left(P=1.9 \times 10^{-6}\right)$, hip $\left(P=4.5 \times 10^{-3}\right)$ and hip and/or knee $(P=$ $\left.3.2 \times 10^{-6}\right)$. Variants associated with osteocyte transcriptome signature orthologs explained $\sim 11.6 \%(\mathrm{SE}=0.011)$ of the total estimated SNP heritability of OA at any site. Competitive gene-set analysis ${ }^{62}$ also showed that human orthologs of osteocyte transcriptome signature genes had stronger associations with $\mathrm{OA}$ than non-osteocyte genes (OA at any site $\left(P=9.1 .6 \times 10^{-3}\right)$, at the knee $\left(P=7.2 \times 10^{-3}\right)$, hip $\left(P=3.0 \times 10^{-3}\right)$ and hip and/or knee $\left(P=6.6 \times 10^{-2}\right)$. Enrichment was largely attributable to significant gene-level associations $\left(P<2.6 \times 10^{-6}\right)$ of a small number of osteocyte transcriptome signature orthologs (40/992, $\sim 4 \%$ ) with OA (Fig. 7b and Supplementary Data 11). Mutations in eight of these genes cause monogenic skeletal disorders in humans (7.4-fold enrichment, $P=9.5 \times 10^{-6}$ ) (Supplementary Data 11). In all, 13/40 osteocyte transcriptome signature orthologs were the closest genes to conditionally independent lead OA variants (4fold enrichment, $P=1.2 \times 10^{-5}$ ), indicating osteocyte transcriptome signature genes occur nearest to lead variants more often than expected by chance (Fig. 7a and Supplementary Data 11). Whilst a number of these genes are also expressed in chondrocytes, others such as MEPE, TSKU, SEMA3F, SEMA3G and SEMA7A $\left(P_{\text {JOINT }}<2 \times 10^{-6}\right.$ in all cases, Fig. $\left.7 \mathrm{~b}\right)$ are not ${ }^{65}$, indicating their contribution to OA may at least in part be due to their role in osteocytes. Together, these data show that osteocyte transcriptome signature genes are not only associated with osteoporosis susceptibility, but may also identify genes associated with OA susceptibility.

\section{Discussion}

Osteocytes are critical cellular regulators of the skeleton. To understand the molecular pathways that control the osteocyte network we generated a map of the osteocyte-enriched transcriptome using data derived from long bones at differing anatomical locations, various ages and both sexes and defined an osteocyte transcriptome signature that represents a profile of genes enriched for expression in osteocytes. The majority of these genes have not previously been shown to have a role in bone, including genes that resulted in abnormal structural and functional skeletal phenotypes when deleted in mice. This included novel, non-coding genes, that were restricted in expression to osteocytes, suggesting an additional, unappreciated, level of control over osteocyte function. Integrating this map with 


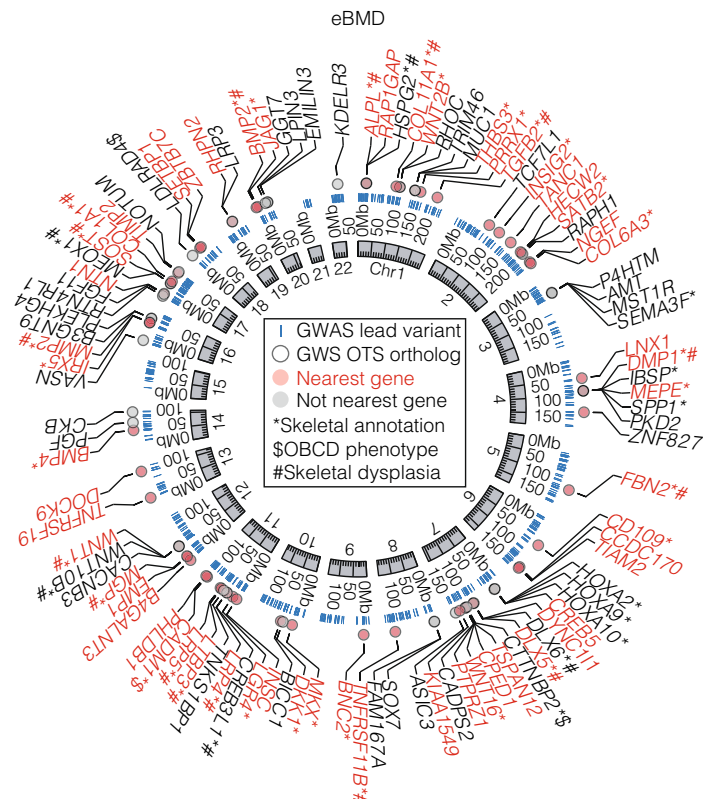

b

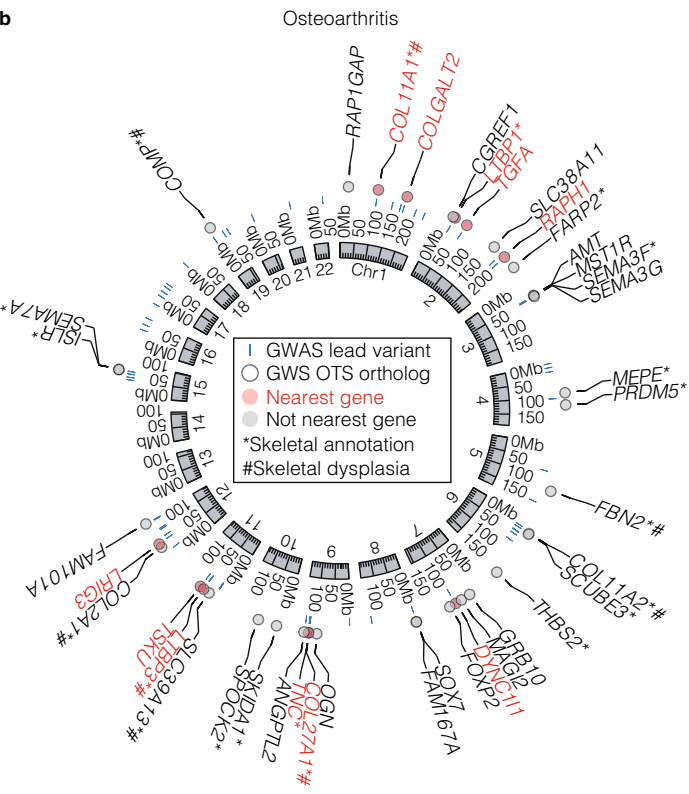

Fig. 7 Osteocyte transcriptome signature genes are associated with eBMD and osteoarthritis in humans. a The top 100 osteocyte transcriptome

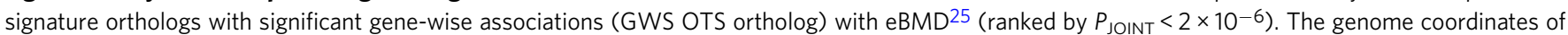
each human ortholog are shown. b. The 40 osteocyte transcriptome signature orthologs with significant gene-wise associations (GWS OTS ortholog) with OA (any subcategory) ${ }^{64}\left(P_{\text {JOINT }}<2 \times 10^{-6}\right)$. Blue bars show the genome coordinates of significant, conditionally independent GWAS variants associated with eBMD (a) or OA (b) (GWAS lead variants). Genes with significant gene-wise associations and that are located nearest to these loci are shown in red, genes that are not nearest to these loci are shown in black. Genes annotated with a role in the skeleton (either GO biological processes database or skeletal phenotype in the MGI database) are denoted with ${ }^{\star}$, genes in the Origins of Bone and Cartilage Disease (OBCD) database are denoted with $\$$ and genes that cause skeletal genetic disorder when mutated in humans denoted with \#.

orthogonal gene expression datasets $13,15,16,28,50$, functional skeletal phenotyping data ${ }^{66}$, GWAS datasets ${ }^{25,64}$, and the nosology of genetic skeletal disorders ${ }^{21}$, provided new understanding of the fundamental role of the osteocyte in skeletal health and disease.

First, we showed the osteocyte transcriptome is conserved among bones from different anatomical locations, yet Homeobox genes demarcate osteocytes obtained from upper and lower limbs. This indicates that the molecular postcode in osteocytes established during embryonic limb patterning persists in the adult osteocyte, suggesting that their function requires information on their anatomical location. In support of this, site-specific patterns of Homeobox genes in adult skeletal progenitors and cells lining bone have been implicated in bone repair $^{67,68}$, and the regulation of bone mass ${ }^{69}$. Second, we demonstrate the osteocyte transcriptome is regulated differentially between the sexes but only in adulthood, suggesting that osteocyte function during skeletal growth and development is similar in males and females. The adult sexual dimorphism involves genes that control perilacunar remodelling, a process associated with rapid calcium release from the skeleton during lactation, and glucocorticoid-induced bone loss ${ }^{15,37,70}$. This expands our understanding of the genes that control this pivotal process. Third, the osteocyte transcriptome signature is enriched with genes that control axonal guidance and neuronal network formation, which are up-regulated early in osteocyte differentiation. The remarkable physical and molecular similarity between the osteocyte network and neuronal networks indicates that osteocytes have repurposed neuronal molecular control pathways to facilitate osteocyte network formation and function. Leveraging knowledge from neuronal networks is likely to accelerate understanding of how the osteocyte network forms and functions at a molecular level.
An important challenge facing genome-wide association studies of skeletal diseases is how to map genome-wide significant variants reliably to their causal genes. Integrative analysis of the osteocyte transcriptome signature with human genetic association studies of osteoporosis identified new candidate causal genes that may be associated with skeletal disease susceptibility. These included AUTS2, CTTNBP2 and LDLRAD4, whose deletion in mice results in abnormal skeletal phenotypes. AUTS2 and CTTNBP2 are regulators of neuronal cell migration and branching 52,56, further highlighting the importance of neuronlike pathways in osteocyte network function and in the control of the skeleton. This strategy also identified new candidate loci associated with $\mathrm{OA}$, suggesting that expression of these genes in osteocytes may contribute to the remodelling of subchondral bone, which is critical in the pathogenesis of $\mathrm{OA}^{71}$. In addition to complex diseases, analysis of the osteocyte transcriptome signature also revealed that genes known to cause monogenic skeletal disorders are enriched in osteocytes. Enrichment was most striking among the osteogenesis imperfecta and decreased bone density group, which is a group characterised by bone fragility. Indeed, the majority of the genes that cause OI are found in the osteocyte transcriptome signature. These genes are involved in matrix synthesis and transcript levels were as high or in some cases higher than osteoblasts or bone-lining cells in the orthogonal dataset analysis. Whilst these genes are actively transcribed in osteocytes they may not translated until required, for example during perilacunar remodelling. Moreover, these results suggest that, in addition to the canonical model of OI as a disease of osteoblasts, the pathogenesis of OI may also crucially involve the osteocyte network. This conclusion is supported by studies showing the osteocyte network is dysregulated in patients with $\mathrm{OI}^{72,73}$. Together this illustrates that linking knowledge of the osteocyte transcriptome signature and functional phenotyping in 
mice, with GWAS and/or the nosology of genetic skeletal disorders, identifies genes associated with skeletal disease in humans and helps prioritise genes for further analysis.

This study has limitations. The bone samples investigated are long bones and we have not included spine or calvaria. Samples include both cortical and cancellous bone, although cancellous bone represented only $1-2 \%$ of the total bone tissue sampled, suggesting the transcriptome data mainly represents genes transcribed in cortical osteocytes. Furthermore, we cannot exclude the possibility that contaminating cells may contribute to the osteocyte-enriched transcriptome, although careful tissue processing and validation using orthogonal datasets strongly suggests the dataset is restricted to genes that are enriched in osteocytes and highly unlikely to include genes expressed only in minor populations of non-osteocyte cells. Lastly, although this study has analysed the trancriptome of osteocytes it has not determined protein expression, nevertheless, skeletal phenotyping of mice with deletions of identified genes have significant skeletal phenotypes suggesting they are translated and functionally important in the skeleton.

Diseases affecting skeletal development, maintenance and repair result in a considerable health burden ${ }^{74}$ and provide the imperative to understand the pivotal role of osteocytes in skeletal physiology and pathophysiology. The osteocyte-enriched transcriptome map and osteocyte transcriptome signature, reported here, provide major new insights into the genes and molecular pathways that regulate osteocyte differentiation, osteocyte network formation and mature osteocyte function and are highly enriched for genes implicated in rare and common polygenetic skeletal disease. Thus, defining the osteocyte transcriptome signature represents a critical step forward in understanding the fundamental processes underlying skeletal physiology and the cellular and molecular aetiology of human skeletal disease.

\section{Methods}

Transcriptome sequencing mouse cohorts. Transcriptome sequencing and morphological analyses were performed on wild-type, immune-competent, C57BL6/NTac mice. The Garvan/St Vincent's Animal Ethics Committee approved all animal experiments (Protocol ID 16/01 and 12/44). Mice were maintained in a specific pathogen-free facility and group-housed ( $2-5$ animals per cage) with continuous access to food and water. None of the mice had noticeable health or immune status abnormalities, and were not subject to prior procedures. Three experimental cohorts were used:

Bone comparison cohort. Bone samples were collected from left and right tibiae, femora and humeri of eight 16-week-old male C57BL6/NTac mice as detailed in the Sample collection and in situ osteocyte isolation section below ( $n=16$ per bone type, 48 samples total). From each mouse, all samples were collected and processed within 20 minutes of killing. Histology and $\mu \mathrm{CT}$ analysis were performed on all samples collected from the right limbs as detailed in the Morphological analysis of bone samples section below ( $n=8$ per bone type, 24 samples total). Bones from the left limbs were processed to obtain in situ isolated osteocytes as detailed in the Sample collection and in situ osteocyte isolation section below. Transcriptome sequencing was performed on all samples collected from the left limbs as detailed in the RNA extraction, transcriptome library preparation and RNA-sequencing section below ( $n=8$ per bone type, 24 samples total). Samples were sequenced to an average depth of $\sim 30$ million reads per sample.

Skeletal maturation cohort. Left and right humeri were collected from 4, 10, 16 and 26-week-old female and male C57BL6/NTac mice ( $n=5$ per sample type, 80 samples in total). Breeding was stratified so samples from each age could be collected within a single 36-h time period. Samples were collected in groups of eight mice (one from each time point in each sex) to avoid confounding batch effects. All samples were collected within 15 min of killing. Intact bones from the right limb were used for morphological analysis by dual energy X-ray absorptiometry (DXA) as detailed in the Morphological analysis of bone samples section below. Transcriptome sequencing was performed on all samples collected from the left limb as detailed in the RNA extraction, transcriptome library preparation and RNA-sequencing section below ( $n=5$ per bone type, 40 samples total). Samples were sequenced to an average depth of $\sim 25$ million reads per sample.
Osteocyte enrichment cohort. Left and right humeri were collected from five 10week-old male C57BL6/NTac mice ( $n=5$ per sample type, 10 samples total). Al samples were collected and processed within 20 min of killing. Bones from the left limb were processed to obtain in situ isolated osteocytes as detailed in the Sample collection and in situ osteocyte isolation section below. Bones from the right limb were processed in an identical manner but not flushed with PBS or centrifuged so as to retain the bone marrow. Transcriptome sequencing was performed on all samples as detailed in the RNA extraction, transcriptome library preparation and RNA-sequencing section below ( $n=5$ per sample type, 10 samples total). Samples were sequenced to an average depth of $\sim 20$ million reads per sample.

Sample collection and in situ osteocyte isolation. Mice were killed by $\mathrm{CO}_{2}$ asphyxiation and cervical dislocation. To isolate osteocytes within the bone samples, soft tissue including muscle, ligaments, tendon and periosteum were removed. Diaphyseal bone from the tibia was isolated cutting at the fibula junction and then $1 \mathrm{~mm}$ distal to the proximal and distal growth plates, and from the femur by cutting the bone immediately proximal to the third trochanter and then $1 \mathrm{~mm}$ proximal to the distal growth plate. The humeri were cut immediately proximal to the deltoid tuberosity and then $1 \mathrm{~mm}$ proximal to the epicondyles before completely removing the deltoid tuberosity along the bone shaft. Bone marrow from each bone was removed by first flushing with PBS until visibly clean and then centrifugation at $14,000 \times g$ for $15 \mathrm{~s}$. Bones were cut into pieces and snap frozen in liquid $\mathrm{N}_{2}$ for storage.

\section{Morphological analysis of bone samples}

Dual energy X-ray absorptiometry. To examine changes in bone structure in the Skeletal maturation cohort, bones were scanned by DXA. Whole femoral length, bone mineral density (BMD) and bone mineral content (BMC) were measured in excised left femora using a Lunar Piximus II dual X-ray absorptiometer (DXA) (GE Medical Systems). Femora were scanned with tibiae attached and the knee joint in flexion to ninety degrees to ensure consistent placement and scan of the sagittal profile.

Micro-CT $(\mu C T)$ visualisation of bone samples. Bones were scanned using a Skyscan Model 1172 microCT scanner (Bruker) at $50 \mathrm{kV}, 200 \mathrm{~mA}$ with a $0.5-\mathrm{mm}$ aluminium filter at a pixel size of $4.3 \mu \mathrm{m}$. Images were captured every $0.4^{\circ}$ through $180^{\circ}$ reconstructed and cortical and cancellous bone regions were defined and bone volume analysed using NRecon and CTAn software (Bruker, http://bruker-microct. com/products/downloads.htm). Three-dimensional models were created using the Drishti-2 tool ${ }^{75}$ (https://github.com/nci/drishti).

Histology. Histological analysis was performed on bone samples from the Bone Comparison Cohort to establish the efficacy of the osteocyte isolation methodology. Samples were decalcified in $0.5 \mathrm{M}$ EDTA at $37^{\circ} \mathrm{C}$ for 24 hours and embedded in paraffin. In total, $3 \mu \mathrm{m}$ sections (parasagittal plane) were cut on a RM2265 microtome (Leica), mounted on superfrost plus (Thermo Fisher Scientific, 4951PLUS4) and stained with Mayer's hematoxylin and eosin (Sigma, MHS1). Images of each section were captured using $\times 10$ and $\times 20$ objectives with an Aperio Scanscope slide scanner (Leica) and processed by Aperio Imagescope (Leica, https://www.leicabiosystems.com/digital-pathology/manage/aperio-imagescope) and Fiji/ImageJ software ${ }^{76}$ (https://fiji.sc).

Tartrate resistant acid phosphatase staining. EDTA-decalcified bone sections $(3 \mu \mathrm{m})$ were deparaffinized, hydrated and incubated in $1 \mathrm{M}$ Tris-HCl pH9.4 buffer at $37^{\circ} \mathrm{C}$ for $30 \mathrm{~min}$. Sections were then stained for Tartrate Resistant Acid Phosphatase (TRAP) by incubation in $1 \mathrm{M}$ sodium acetate (pH 5.2), Naphtol-ASBIphosphate, and sodium tartrate for $10 \mathrm{~min}$ at $37^{\circ} \mathrm{C}$. Sections were then rinsed in distilled water and counterstained with hematoxylin for $15 \mathrm{~s}$.

Histomorphometry. Histomorphometric assessment of cell-types in cortical bone and on the endocortical surfaces were measured using Osteomeasure software (version 3.2.1.8, Osteometrics Inc). Cell numbers were measured over a 5-mm length of both anterior and posterior endocortical surfaces in each bone sample Measurements began $0.25 \mathrm{~mm}$ from the first field of view below the proximal end of each sample. Bone-cell-types quantified included osteoblasts/bone-lining cells, TRAP positive osteoclasts and osteocytes. Cells that could not be clearly defined as osteocytes, osteoblasts or bone-lining cells were defined as other celltypes.

\section{RNA extraction, transcriptome library preparation and RNA-sequencing}

TRIreagent (Sigma-Aldrich, T9424) was added directly to frozen bone samples and homogenised using a Polytron hand-held homogeniser (PT1200E, Kinematica). RNA was isolated according to the manufacturers protocol and cleaned with an additional ethanol-precipitation step. RNA yield was determined using a Nanodrop (Thermo Fisher Scientific, 2000) and RNA integrity determined using the Bioanalyser RNA 6000 Nano Kit (Agilent Technologies, 5067-1511). Total RNA (250 ng) was depleted of ribosomal RNA using RNaseH (Epicentre) and ribosomal RNA targeting oligonucleotides based on a protocol by Adiconis et al., 201377. Briefly, 
total RNA, spiked with External RNA Controls Consortium (ERCC) internal controls (Thermo Fisher Scientific, 4456740), was incubated with ribosomal-RNA targeting oligos (sequences reported in Adiconis et al. ${ }^{77}$ ) and $\mathrm{RNaseH}$ to degrade the rRNAs before the oligos were removed with DNase treatment (Thermo Fisher Scientific, AM2238). RNA was re-purified using magnetic beads according to the manufacturers protocol (Beckman Coulter Genomics, A63987). Total-RNA stranded transcriptome libraries were prepared using the TruSeq Stranded Total RNA LT Sample Prep Kit starting from the fragmentation step in the manufacturers protocol (Illumina, RS-122-2201). Paired-end sequencing ( $2 \times 125$-bp) was carried out on a HiSeq 2500 instrument (Illumina) at the Kinghorn Center for Clinical Genomics, Garvan Institute, Sydney, Australia.

De novo transcriptome assembly and filtering. Sequencing read data was pooled for each bone type in the Bone Comparison Cohort (described above) and de novo transcriptome assembly performed using two different assembly strategies: ab initio, using Trinity ${ }^{78}$, and genome-guided, using Stringtie ${ }^{79}$ Only multi-exon ( $\geq 2$ exons) transcripts assembled by both methods were retained before transcripts assembled in each of the three bones were pooled using Cuffcompare ${ }^{80}$ to generate a non-redundant union set of assembled transcripts. Assembled transcripts with splice patterns matching those in RefSeq ${ }^{81}$ or GENCODE-M5 $5^{82}$ transcriptome annotations were removed to identify novel transcripts. Remaining transcripts were then filtered based on exon length. Briefly, the mean $\log _{2}$-exon-length \pm 2 standard deviations of GENCODE-M5 annotated exons was calculated. Assembled transcripts containing one or more exons outside of this range were removed. The protein coding potential of the remaining transcripts was assessed using CPAT ${ }^{83}$ (http://lilab.research.bcm.edu/cpat). To annotate structures arising from novel loci in the genome, those overlapping known annotated transcripts located on the opposite strand were given a novel_antisense biotype and given gene IDs beginning with Obcda, while transcripts located between known genes were given the novel_intergenic biotype and assigned gene IDs beginning with Obcdi. Novel transcripts for both known and novel genes possess transcript IDs begin with TRINITY. These novel, multi-exon transcripts were then concatenated to the GENCODE-M5 annotation prior to read alignment. Subsequent to this analysis, new gene structures have been predicted. Some contain splice junctions that overlap novel transcripts reported here. In this case, the GENCODE-M13 gene name is contained in brackets beside the unique assigned gene ID

Defining the genes actively expressed in osteocytes. Transcriptome data were trimmed of low-quality reads and adaptor sequences using Trim Galore (https:// github.com/FelixKrueger/TrimGalore) and aligned to the GRCm38.p3 mouse genome, guided by the GENCODE-M5 transcriptome annotation plus the novel assembled transcripts described above, using STAR $^{84}$ and quantified using $\mathrm{RSEM}^{85}$. A threshold of gene activity was calculated based on the bimodal distribution of normalised gene expression as described in Hart et $\mathrm{al}^{27}$. Briefly, FPKM values were $\log _{2}$-normalised (nFPKM), omitting genes with $\mathrm{FPKM}=0$. The kernal density estimate (KDE) of these values was then calculated (using Scotts rule of thumb for bandwidth) and the maximum KDE value determined. A Gaussian distribution was then fitted, with the mean $(\mu)$ at the KDE maximum and the standard deviation (SD) based on normalised expression values greater than $\mu$. The nFPKM values were then transformed to zFPKM using zFPKM $=$ $(\mathrm{nFPKM}-\mu) / \mathrm{SD}$. Active expression in a sample was defined as those with $>-2.6$ zFPKM, the conservative range suggested in the original publication ${ }^{27}$. This generated sample-specific thresholds for gene activity which are listed in Supplementary Data 1. Genes were considered actively expressed in a given bone sample type if they were above the sample-specific gene activity threshold in all biological replicates. The numbers of active genes in each bone type were classed according to the gene biotype defined in the GENCODE-M5 transcriptome annotation.

Orthogonal validation of gene expression in osteocytes. To validate the active expression of genes in the osteocyte network and to ensure the genes identified were not derived from minor populations of non-osteocytic contaminating cells we used 3 publicly available datasets;

Osteocytic cell-line. This transcriptome sequencing dataset profiled data from the IDG-SW3 mouse osteocyte cell line, an in vitro model of osteoblast-like cell to osteocyte differentiation ${ }^{50}$ (ArrayExpress accession E-GEOD-54783 https://www. ebi.ac.uk/arrayexpress/experiments/E-GEOD-54783). These data were originally published as part of a temporal study of osteocyte differentiation. Data from days 3 , 14 and 35 represent osteoblast, early osteocyte and mature osteocyte stages, respectively ${ }^{86}$. Raw data were aligned and quantified using the GENCODE-M5 transcriptome annotation plus the novel assembled transcripts. Genes were considered to be expressed in this dataset if they had a read count $\geq 10$ in each replicate $(n=3)$ in every replicate of early or mature osteocytes.

Laser-capture micro-dissected osteocytes. This microarray dataset profiled gene expression in laser-capture micro-dissected osteoblasts, bone-lining cells and osteocytes $^{87}$ (GEO accession GSE71306). These data were originally published as part of an investigation into the bone-cell response to Sclerostin-antibody treatment. Only untreated control samples were used in this analysis. As this dataset was generated from rat bone, mouse-orthologs of rat genes were identified using the biomaRt package ${ }^{88}$. Background expression and control probes were filtered from the data and mean signal intensity calculated for duplicate probes corresponding to a single gene using the oligo and affycoretools packages ${ }^{89,90}$. Genes present in each sample were determined using the Wilcoxon signed rank-based gene expression detection algorithm (MAS5calls function from the affy package ${ }^{91}$ ). Genes were considered to be expressed in this dataset if they were present in more than $50 \%$ of osteocyte samples $(>20 / 40)$.

Collagenase-digested osteocytes. This microarray dataset profiled gene expression in osteocytes from primary bone tissue, with cells removed from the bone surface by flushing, centrifugation and serial collagenase digestions ${ }^{15}$ (ArrayExpress accession E-GEOD-23496 https://www.ebi.ac.uk/arrayexpress/experiments/E-GEOD-23496) This dataset was originally published in a study investigating gene expression changes in the osteocyte network in virgin mice, mice during lactation and mice post lactation. Background expression and control probes were filtered from the data and mean signal intensity calculated for duplicate probes corresponding to a single gene using the oligo and affycoretools packages ${ }^{89,90}$. Genes present in each sample were determined using the Wilcoxon signed rank-based gene expression detection algorithm (MAS5calls function from the affy package ${ }^{91}$ ). Genes were considered to be expressed in this dataset if they were detected in all replicates $(n=3)$ of any experimental condition.

\section{Analysis of the osteocyte transcriptome between skeletal sites}

Gene activity. Gene activity in the Bone Comparison Cohort was defined as per the methods section Defining the genes actively expressed in osteocytes. Genes expressed above the sample-specific activity threshold in $8 / 8$ replicates of either tibiae, femora or humeri were considered active. Active genes were classed according to gene biotype defined in the GENCODE-M5 transcriptome annotation.

Correlation. The Pearson correlation between each sample was calculated based on normalised counts of active genes in any bone type and plotted using the ggplot2 package ${ }^{92}$. The mean correlation between samples for each bone type comparison is also reported.

Differential expression. Differential gene expression analysis between bones was performed using the limma package ${ }^{93}$ on the voom-normalised ${ }^{94}$ counts of active genes in the Bone comparison cohort. The topTreat function identified differentially expressed genes with evidence of a log-fold change (LFC) $>0.5$ between bone types with a false discovery rate (FDR) corrected $P<0.05$. Genes with expression restricted to specific skeletal sites were active in at least one bone type (above the sample-specific activity threshold in $8 / 8$ replicates of a given bone type) and inactive in another bone type (below the sample-specific activity threshold in $8 / 8$ biological replicates).

\section{Comparison with other organs and tissues}

Gene activity. To compare the osteocyte transcriptome with transcriptomes from other tissues, sequencing read data were obtained from Zhang et al. ${ }^{28}$ (ArrayExpress accession E-GEOD-54652 https://www.ebi.ac.uk/arrayexpress/experiments/ E-GEOD-54652). Gene activity was defined as per the methods section Defining the genes actively expressed in osteocytes. This dataset contains 8 replicates of 12 tissues types collected for a single study under controlled conditions. Genes expressed above the sample-specific activity threshold in $8 / 8$ replicates of either the adrenal gland, aorta, brown fat, brainstem, cerebellum, heart, hypothalamus, kidney, liver, lung, muscle or white fat were considered active. This identified the active genes in 12 non-skeletal organs and tissues.

PCA. To compare the transcriptome of osteocytes and other tissues, FPKM of all genes active in any tissue type were quantile-normalised and scaled before principal components (PCs) were calculated and used to cluster samples. Fitting of 95\% CI ellipses and plotting of samples using the first two PCs was performed with ggplot2.

Gene specificity. To examine the specificity of gene expression in osteocytes relative to these other tissues we used the Tau specificity index ${ }^{29}$. Briefly, median $\log _{2}$ FPKM values were calculated for each tissue for all genes actively expressed in the osteocyte transcriptome (pooling samples from the three skeletal sites to get a single median value for osteocytes). These values were quantile-normalised and Tau calculated for each gene in each tissue using the tispec R-package (https://rdrr.io/github/roonysgalbi/tispec). Genes were classified as either lowspecificity $(\mathrm{Tau}<0.15)$, moderate-specificity $(0.15 \leq \mathrm{Tau} \leq 0.85)$ or high specificity (Tau $>0.85$ ). The density distribution of Tau in each tissue was plotted with ggplot2, using default axis scaling to ensure visualisation of density distribution in each tissue. 


\section{Defining changes in the osteocyte transcriptome with skeletal maturation} Gene activity. Gene activity in the Skeletal Maturation Cohort was defined per the methods section Defining the genes actively expressed in osteocytes. Genes expressed above the sample-specific activity threshold in $5 / 5$ replicates of any age in either sex were considered active. Active genes were classed according to gene biotype defined in the GENCODE-M5 transcriptome annotation.

PCA. PCA was performed on the Skeletal Maturation Cohort between female samples (any age), male samples (any age) and sexes at each age (4, 10, 16 and 26weeks-old). For each comparison, PCs were calculated based on the scaled-FPKMs of genes active in any sample type, with the first two principal components then used to cluster samples. Significant differences between centroids was determined by Hotellings t-test. Fitting of 50\% CI ellipses and plotting was performed using the ggplot2 R-package.

Weighted gene co-expression network analysis (WGCNA). To identify clusters of genes with highly correlated patterns of gene expression during skeletal maturation, WGCNA was performed on the normalised counts of genes expressed in either sex at any age $\left(4,10,16\right.$ or 26 -weeks-old) using the WGCNA package ${ }^{95}$. Briefly, genewise connectedness was calculated using the bi-weight mid-correlation function (bicor) across all 40 samples in the Skeletal Maturation Cohort. A soft-thresholding power of eight was calculated as gene connectedness resembled a scale-free network (the scale-free topology model fit $R>0.9$ ). Next, a weighted, signed network adjacency matrix was calculated, raising the gene-wise correlation coefficient to the soft-thresholding power with a $10 \%$ outlier threshold (maxPOutliers $=0.1)$. A topological overlap matrix was constructed based on network adjacency and calculated matrix dissimilarity. Hierarchical clustering was performed on the dissimilarity matrix to group genes based on their connectedness and clusters of highly connected genes identified using the hybrid cutreeDynamic R-function 96 . Clusters with correlated patterns of expression were merged (cut-height $=0.25$ ) leaving 7 clusters (denoted by colours as per WGCNA convention) of highly connected genes with distinct patterns of expression. Genes that were not correlated with each other or with genes in other clusters were allocated to an 8th Grey cluster.

Cluster characterisation. Clusters with expression patterns significantly associated with variation in age, sex or both age and sex were then identified. Briefly, the pattern of gene expression within each WGCNA cluster were summarised into eigengene values $\left(\mathrm{EV}_{\text {cluster }}\right)$, defined as the first $\mathrm{PC}$ of cluster gene expression variance. Line plots produced with Prism (GraphPad v7, https://www.graphpad. $\mathrm{com} /$ scientific-software/prism) were used to visualise the mean and SD of EV in male and female mice. Three linear models were fitted to each cluster $\left(\mathrm{EV}_{\text {cluster }}\right.$ Age, $\mathrm{EV}_{\text {cluster }} \sim$ Sex, $\mathrm{EV}_{\text {cluster }} \sim$ Age + Sex + Age $\times$ Sex) using the lm function of the stats R-package. The goodness-of-fit of each model was tested with the Bayesian Information Criterion (BIC), with the lowest value across the three models taken to be the optimum for each cluster. The adjusted- $\mathrm{R}^{2}\left(\right.$ adj- $\left.\mathrm{R}^{2}\right)$ calculated by the $\mathrm{lm}$ function was used to evaluate the strength of each models' association with EV, with adj- $R^{2}>0.6$ considered a strong association. For genes in each cluster, expression in osteocytes was validated in independent, orthogonal datasets, as per method section Orthogonal validation of gene expression in osteocytes. Significantly enriched Gene Ontology (GO) biological processes ${ }^{40}$, Kyoto Encyclopedia of Genes and Genomes (KEGG) pathways ${ }^{97}$ and Disease Ontology (DO) terms $^{98}$ in each cluster were identified using the ClusterProfiler and DOSE packages $^{99,100}$ (Bonferroni-corrected $P<0.05$ ).

Analysis of Magenta cluster genes. Heatmaps of Magenta Cluster gene expression and line plots of selected genes (top 20 most strongly correlated with $\mathrm{EV}_{\text {Magenta) }}$ ) were generated using the mean of scaled and normalised gene expression counts, calculated across all ages in both sexes. Heatmaps were produced using the gplots package ${ }^{101}$ and line plots produced with Prism (GraphPad v7). Semantically similar GO terms were identified within the GO biological processes significantly enriched in the Magenta Cluster (Bonferroni-corrected $P<0.05$ ) using the ReViGO webtool $^{102}$ (http://revigo.irb.hr/). Briefly, redundant GO terms (similarity >0.9) were removed and multidimensional scaling (MDS) coordinates calculated based on the SimRel semantic similarity algorithm. MDS coordinates were then used to identify clusters of semantically similar GO terms using the mclust package ${ }^{103}$. The optimum number of clusters (4) was selected among models with unequal variance using the Bayesian Information Criterion (BIC). Bar plots were produced with ggplot2. Expression of top-ranked Magenta genes in osteocytes was assessed using orthogonal datasets described in method section Orthogonal validation of gene expression in osteocytes. To determine whether the Magenta Cluster identified genes associated with perilacunar remodelling, their expression was examined during lactation in the Collagenase-digested osteocytes microarray datase ${ }^{15}$ (ArrayExpress accession https://www.ebi.ac.uk/arrayexpress/experiments/EGEOD-23496). Eighty-four of the 95 Magenta Cluster genes were represented on the microarray. Competitive gene set testing accounting for inter-gene correlation was performed on each skeletal maturation cluster using the CAMERA function of the limma package ${ }^{104}$. Tukey boxplots of gene expression during lactation were generated for each cluster based on the mean zscores of normalised probe intensity, calculated across all conditions.
Identification of osteocyte-enriched genes. Genes enriched in osteocytes were identified by comparing gene-counts between the samples of isolated osteocytes and samples retaining bone marrow, described in the Osteocyte enrichment cohort. Gene activity in the Osteocyte enrichment cohort was defined as per the methods section Defining the genes actively expressed in osteocytes. Genes expressed above the sample-specific activity threshold in 5/5 replicates of either condition were considered active. Counts for active genes were normalised by library size only and the gene-wise $\log _{2}$-fold change (LFC) in normalised read count was calculated between conditions. The density distribution of LFC values was calculated using Scotts rule of thumb for bandwidth. This was plotted to reveal multiple local maxima at different levels of osteocyte enrichment. Genes were grouped within these component populations using a Gaussian Mixture Model (GMM) that was fitted to the LFC density distribution between conditions. The optimum number of components (4) was determined using the BIC among models with unequal variance. K-means clustering $(k=4)$ was then used to determine initiation parameters for Expectation-Maximization fitting of the four component GMM using the mixtools R-package ${ }^{105}$. Gene ontology analysis was performed on the top 1000 genes in each cluster, as ranked by posterior probability, using the ClusterProfiler R-package ${ }^{99}$. An enrichment threshold was calculated at two standard deviations above the mean LFC of the second most enriched GMM component (Component 2 ) to exclude genes likely belonging to suboptimal components. This identified an empirically determined enrichment threshold $=1.63$ LFC. Confidence intervals (95\%-CI) of the mean LFC for individual genes were then calculated. Genes with lower LFC-95\%-CI above the enrichment threshold were deemed significantly enriched in osteocytes. Scatter plots, GMM diagrams and density plots were visualised using ggplot2.

Definition of an osteocyte transcriptome signature. Osteocyte-enriched genes with significantly higher expression in either blood, bone marrow or skeletal muscle relative to osteocyte-enriched bone tissue $(P<0.05)$ were identified using differential gene expression calculations associated with the publicly available data reported by Ayturk et al. ${ }^{13}$. Volcano plots were generated using the ggplot2 Rpackage. The osteocyte transcriptome signature was defined as: (1) Genes actively expressed in osteocytes from any of the Bone comparison cohort, Skeletal maturation cohort or Osteocyte enrichment cohort sample types (detailed in section defining the genes actively expressed in osteocytes) AND (2) Genes enriched for expression in osteocytes relative to bone marrow cells (detailed in section Identification of osteocyte-enriched genes) AND (3) Genes not significantly enriched for expression in blood, bone marrow or muscle relative to bone. The specificity of osteocyte transcriptome signature gene expression relative to 12 other non-skeletal tissues, was determined using the Tau specificity index for all signature genes calculated as per method section Comparison with other organs and tissues. The histogram of signature gene specificity was generated using ggplot2. To compare expression of osteocyte transcriptome signature genes in osteocyte with other bone-cell types we used publicly available microarray dataset which profiled gene expression in laser-capture micro-dissected osteoblasts, bone-lining cells and osteocytes $^{87}$ (GEO accession GSE71306). The design and preprocessing of this dataset is described in method section Orthogonal validation of gene expression in osteocytes - Laser-capture micro-dissected osteocytes. CAMERA gene-set analysis identified significant differences in expression of signature genes between osteocytes and osteoblasts, and between osteocytes and bone-lining cells. Tukey boxplots were generated based on the mean of scaled, normalised probe intensity values for signature genes in each cell-type using ggplot2.

Identification of osteocyte transcriptome signature genes known to affect the skeleton. Osteocyte transcriptome signature genes associated with biological processes important in the skeleton were identified using a curated list of GO biological processes ${ }^{40}$ directly related to the skeleton ${ }^{106}$. Briefly, this list was constructed by filtering GO term descriptions using bone-related keywords. Osteocyte transcriptome signature genes associated with any of the 116 manually curated skeletal biological processes were then identified. Similarly, to identify osteocyte transcriptome signature genes that cause a significant skeletal phenotype when knocked out in mice, a list of mammalian phenotype (MP) terms related to the skeleton was constructed. MP term definitions and descriptions were filtered using bone-related keywords to identify skeletal MP terms. Screening the Mouse Genome Informatics (MGI) database ${ }^{41}$ with skeletal MP terms identified mouse knockout lines with significant skeletal phenotypes (conditional alleles were excluded). Osteocyte transcriptome signature genes associated with a skeletal phenotype when deleted in mice were then identified. Significant over-representation of osteocyte transcriptome signature genes in each of these skeletal gene lists was tested under the hypergeometric distribution $(P<0.05)$.

Osteocyte transcriptome signature enrichment and GO semantic similarity clustering. Significantly over-represented GO biological processes and KEGG pathways in the osteocyte transcriptome signature were identified using the clusterProfiler R-package ${ }^{99}$ (Bonferroni-corrected $P<0.05$ ). Clusters of semantically similar GO biological process in the osteocyte transcriptome signature were identified as described in the section Detailed analysis of Magenta Cluster genes. The optimum number of clusters (8) was selected among models with 
unequal variance using the BIC. Wnt signalling, PTH-signalling and Axonguidance pathway diagrams coloured by gene expression in osteocytes were constructed using output from the Pathview R-package ${ }^{107}$ and data from the KEGG database ${ }^{97}$.

Osteocyte transcriptome signature gene cluster identification during differentiation. To examine the expression of osteocyte transcriptome signature genes during osteocyte differentiation, we analysed publicly available transcriptome sequencing data from an in vitro model of osteoblast-like cell to osteocyte differentiation $^{50}$ (ArrayExpress accession E-GEOD-54783). The design and preprocessing of this dataset is described in method section Orthogonal validation of gene expression in osteocytes - Osteocytic cell-line. Osteocyte transcriptome signature genes were clustered based on their expression in this dataset, with the optimum number of clusters calculated based on the Calinski-Harabasz $(\mathrm{CH})$ index using the clues R-package ${ }^{108}$ and visualised using the heatmap.2 function of the gplots R-package. Tukey boxplots of cluster expression in each differentiation stage were generated based on the mean zscores of normalised gene expression calculated across all conditions using the ggplot2 R-package. Significant enriched GO biological processes associated with genes in each cluster were identified using the ClusterProfiler R-package (Bonferroni adjusted $P<0.05$ ).

Novel osteocyte transcriptome signature gene analysis and deletion in mice. Novel genes were identified based on the de novo transcriptome assembly pipeline described in the De novo transcriptome assembly and filtering section above. The gene structures of novel loci and read-data alignment diagrams were visualised using Gvis ${ }^{109}$, using pooled read data from each bone type. Expression in other tissues was determined using the dataset described in section Comparison with other organs and tissues, with bar plots generated with ggplot2.

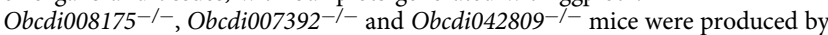
the Mouse Engineering Garvan/ABR (MEGA) Facility (Moss Vale and Sydney, Australia) by CRISPR/Cas9 gene targeting in C57BL/6 J mouse embryos using established molecular and animal husbandry techniques ${ }^{110}$. Experiments performed at the MEGA were approved by the Garvan/St Vincent's Animal Ethics Committee (Protocol ID 18/36). In each case, two single guide RNAs (sgRNAs) were designed to target either side of the genomic DNA encoding the longest predicted transcript and co-injected with polyadenylated Cas9 mRNA into C57BL/ $6 \mathrm{~J}$ zygotes. Microinjected embryos were cultured overnight and introduced into pseudo-pregnant foster mothers. Pups were screened by PCR and Sanger sequencing of ear-punch DNA and founder mice identified that carried deletions including the entire gene sequence. The targeted allele was maintained and bred to homozygosity on a C57BL/6 J background. The details for each line are found in Supplementary Table 1.

Skeletal phenotyping of these lines was performed as described below (section Structural and functional skeletal phenotype analysis of mice with deletions of osteocyte transcriptome signature genes) except that digital X-ray images were recorded at a $10-\mu \mathrm{m}$ resolution using a Faxitron UltraFocus (Faxitron Bioptics LLC,Tucson, Arizona USA) operating in full manual mode at $22 \mathrm{kV}$.

Structural and functional skeletal phenotype analysis of mice with deletions of osteocyte transcriptome signature genes. The Origins of Bone and Cartilage Disease (OBCD) programme ${ }^{66}$ is undertaking a validated rapid-throughput multiparameter skeletal phenotype screen of mutant mouse lines generated by the Wellcome Trust Sanger Institute as part of the International Mouse Phenotyping Consortium (IMPC) ${ }^{111}$ and International Mouse Knockout Consortium (IMKC) ${ }^{112}$ effort. The OBCD mouse studies were undertaken by Wellcome Trust Sanger Institute Mouse Genetics Project (MGP) as part of the IMPC. This was licensed by the UK Home Office (PPLs 80/2485 and P77453634) in accordance with the 1986 Animals (Scientific Procedures) Act and approved by the Wellcome Sanger Institute's Animal Welfare and Ethical Review Body.

All mice generated by the MGP were produced ( $\mathrm{Tm} 1$ alleles ${ }^{113}$, CRISPR alleles $^{114}$ ) and maintained on a C57BL/6NTac background. Mice were fed either a Breeder's Chow (Mouse Breeder Diet 5021, 21\% kcal as fat, Labdiet, London, UK) or a high fat diet (Western RD, 82,9100, Special Diet Services, Witham, UK) from 4 weeks of age. Prior to tissue collection, mice underwent a standardised broad primary phenotype screen (https://www.mousephenotype.org/impress/ PipelineInfo?id=15) ${ }^{115}$. Detailed OBCD phenotyping methods were then performed as below:

Origins of bone and cartilage disease phenotyping methods. Left lower limb and tail samples from 16-week-old female wild-type and knockout mice were stored in $70 \%$ ethanol at $4{ }^{\circ} \mathrm{C}$, anonymized and randomly assigned to batches for rapidthroughput analysis in an unselected fashion ( $n=2-6$ per line). Overall, 19 structural and functional skeletal parameters were determined for femur and vertebrae samples from each mouse studied and compared to reference data (shown as a range with mean and 1 and 2 standard deviations) obtained from 320 16-week-old wild-type C57BL/6NTac female mice and collected in batches from across the study time-course. Coefficients of variation for each skeletal parameter were: femur BMC (2.0\%) and length (2.1\%); vertebra BMC (2.1\%) and length (2.3\%); trabecular bone volume/tissue volume (18.5\%), trabecular number $(7.3 \%)$, trabecular thickness (7.9\%) and trabecular spacing (8.3\%); cortical bone thickness (4.3\%), internal diameter (6.0\%) and BMD (4.0\%); femur yield load (13.2\%), maximum load (10.0\%), fracture load (29.0\%), stiffness (13.7\%) and energy dissipated before fracture (26.7\%); and vertebra yield load (13.0\%), maximum load (10.3\%) and stiffness (13.3\%).

Digital $x$-ray microradiography. Soft tissue was removed from skeletal samples and digital X-ray images were recorded at a $10-\mu \mathrm{m}$ resolution using a Faxitron MX20 operating at $26 \mathrm{kV}$ (Qados, Cross Technologies plc, Sandhurst, Berkshire, UK). Cleaned lower limb and caudal vertebrae 6 and 7 were imaged together with $1 \mathrm{~mm}$ diameter steel, aluminium and polyester standards. Bone lengths and relative bone mineral content (BMC) were determined as previously described ${ }^{66}$. Briefly, femur length and mean length of caudal vertebrae 6 and 7 were determined using ImageJ and images calibrated using an X-ray image of a digital micrometre. To determine relative BMC, $2368 \times 234016$-bit DICOM images were converted to 8 -bit Tiff images in ImageJ, the grey levels of the polyester and steel standards were defined and the image stretched between the polyester (grey level 0) and steel (grey level 255) standards. Increasing gradations of mineralisation density were represented in 16 equal intervals by applying a pseudocolour lookup table to each image. For each sample the median grey level (0-255) of the femur and caudal vertebrae 6 and 7 was calculated.

Micro-CT analysis. Cortical and trabecular parameters were determined by microCT using a Scanco $\mu$ CT50 (Scanco medical, Zurich, Switzerland). Samples were scanned at $70 \mathrm{kV}, 200 \mu \mathrm{A}$, with a $0.5-\mathrm{mm}$ aluminium filter, $1 \mathrm{~s}$ integration time, no averaging, and images captured every $0.36^{\circ}$ though $180^{\circ}$ rotation. Reconstructions, ROI selection and analyses were performed using Scanco software. Trabecular bone parameters (trabecular bone volume as a percentage of tissue volume BV/TV, trabecular number Tb.N, trabecular thickness $\mathrm{Tb}$.Th and trabecular spacing $\mathrm{Tb} . \mathrm{Sp}$ ) were calculated from scans at a voxel resolution of $5 \mu \mathrm{m}$ in a 1-mm-long region of the trabecular compartment beginning $100 \mu \mathrm{m}$ proximal to the distal femoral growth plate. Cortical bone parameters (cortical thickness Ct.Th, internal endosteal diameter, and BMD) were calculated from scans at voxel resolution of $10 \mu \mathrm{m}$ from a $1.5 \mathrm{~mm}$ long region of mid-shaft cortical bone centred $56 \%$ along the length of the femur distal to the femoral head.

Micro-CT analysis of osteocyte lacunae. Osteocyte lacuna parameters were determined by micro-CT using a Scanco $\mu \mathrm{CT} 50$ as described above. Osteocyte parameters (lacuna number Lc.N, lacuna volume Lc.V and lacuna sphericity Lc.Sph) were calculated from scans at a voxel resolution of $1 \mu \mathrm{m}$ from a $0.25-\mathrm{mm}$-long region of mid-shaft cortical bone centred $60 \%$ along the length of the tibial from the tibial plateau. The micro-CT segmentation threshold was optimised by analysing lacuna area (Lc.Ar and Lc.N) in longitudinal sections of cortical bone imaged by both back scattered-election scanning-electron microscopy (considered the gold standard) and micro-CT. Micro-CT data was reconstructed using a range of segmentation thresholds and the distribution of Lc.Ar and Lc.N did not differ between BSE-SEM and micro-CT at a segmentation threshold of 350 (equivalent to 851.7 $\mathrm{mg} \mathrm{HAcm}{ }^{-3}$ ). Therefore, a segmentation threshold of 350 was subsequently used for the analysis of all samples. DICOM images were processed using Fiji ${ }^{76}$ and lacunae rendered using its Volume Viewer (https://imagej.nih.gov/ij/plugins/ volume-viewer.html). Lc.V was determined using BoneJ Particle Analyzer ${ }^{116}$ and L Sph using Fiji 3D Shape Measure ${ }^{117}$. Following determination of Lc.N and Lc.N/ BV for each sample, the maximum equivalent number of lacunae were randomly selected from each sample (2693) and the volume and sphericity distribution for each mutant was compared to WT using Kruskall-Wallis analysis (GraphPad Prism v8). Differences between mutant and WT were considered valid if significant in all 10 permutations performed.

Biomechanical testing. Destructive 3-point bend tests and compression tests were performed on an Instron 5543 materials testing load frame (Instron Limited, High Wycombe, UK). Femur strength and toughness (yield load, maximum load, fracture load, stiffness, \% energy dissipated prior to fracture) were derived from destructive three-point bend testing using a $50-\mathrm{N}$ load cell and custom mounts with rounded supports to reduce cutting and shear loads. Bones were positioned horizontally with the anterior surface upwards between two mounting points with a span of $8 \mathrm{~mm}$. Load was applied vertically to the mid-shaft with a constant rate of displacement of $0.03 \mathrm{~mm} / \mathrm{second}$ until fracture. The biomechanical properties of caudal vertebrae 6 and 7 (yield load, maximum load and stiffness) were derived from compression testing using a 500-N load cell and two custom anvils. Vertebrae were bonded in vertical alignment to a custom anvil support using cyanoacrylate glue and load was applied vertically at a constant rate of displacement of $0.03 \mathrm{~mm} / \mathrm{s}$ and a sample rate of $20 \mathrm{~Hz}$ until approximately $1 \mathrm{~mm}$ of displacement had occurred $^{66}$.

Tissue expression screen. Expression in other non-skeletal organs tissues of Auts2, Dact3, Ldlrad4 and Cttnbp2 and genes with established role in osteocyte biology ${ }^{38}$ was analysed using data described in method section Comparison with other organs and tissues. The mean expression in each tissue was first calculated and then the percentage of this mean relative to maximum mean expression across 
all tissues used to construct heatmaps with the ggplot2 package. Bar plots of normalised expression (FPKM) were constructed with ggplot2.

\section{Association of osteocyte transcriptome signature genes with genetic skeletal} disorders. Osteocyte transcriptome signature gene orthologs known to cause rare skeletal genetic disorders in humans were identified in the Nosology and Classification of Genetic Skeletal Disorders ${ }^{21}$. Significant over-representation was examined under the hypergeometric distribution, using the parameters:

- Population - the number of mouse genes with human orthologs actively expressed in any of the Bone comparison cohort, Skeletal maturation cohort or Osteocyte enrichment cohorts or any of the 12 tissues described in the Comparison with other organs and tissues section (15,368 genes)

- Successes in population - the number of genes known to cause genetic skeletal disorders (either total or within each nosology-defined skeletal disease groups) within the population (432 genes)

- Sample size - the number of osteocyte transcriptome signature genes within the population(1043 genes)

- Successes in sample - the number of osteocyte transcriptome signature genes known to cause genetic skeletal disorders(either total or within each individual nosology-defined skeletal disease groups) within the population (90 genes)

Significant enrichment of osteocyte transcriptome signature gene orthologs among all genes in the nosology, and within each of the nosology-defined skeletal disease groups was calculated using the Graeber lab online hypergeometric calculator (https:// systems.crump.ucla.edu/hypergeometric/index.php). Donut plots, bubble plots and bar charts were constructed using ggplot2. Genes known to cause osteogenesis imperfecta (OI genes) were identified ${ }^{57}$ with the addition of $S E C 24 D^{59}$ and $F A M 46 A^{58}$. OI-gene expression during osteocyte differentiation was assessed using an independent, orthogonal dataset described in method section Orthogonal validation of gene expression in osteocytes - Osteocytic cell-line. OI-gene expression in osteocytes relative to other bone-cell types was assessed with an independent, orthogonal dataset described in Methods section Orthogonal validation of gene expression in osteocytes - Laser-capture micro-dissected osteocytes. Significant differences in gene expression between cell types were calculated by two-way ANOVA (adjusted for multiple-comparisons) and visualised using GraphPad Prism v7. Expression in other non-skeletal organs tissues was analysed using data described in method section Comparison with other organs and tissues. The mean expression in each tissue was first calculated and then the percentage of this mean relative to maximum mean expression across all tissues used to construct heatmaps with the ggplot2 R-package. OI gene expression during skeletal maturation was analysed using data described in section Defining changes in the osteocyte transcriptome with skeletal maturation. The strength and significance of Pearson correlation between OI gene expression and age were calculated for each sex using the cor,test function in the stats R-package. Lineplots were produced in GraphPad Prism v7.

\section{Enrichment of osteocyte transcriptome signature for orthologs associated} with osteoporosis and OA. Competitive gene-set analysis, LDSC-SEG, and analysis of genes nearest to significant loci, were used to investigate whether human osteocyte transcriptome signature orthologs associated with: (i) quantitative ultrasound-derived heel bone mineral density (eBMD), a predictor of osteoporosis susceptibility, and (ii) self-reported, or hospital-diagnosed osteoarthritis.

Datasets used for analysis. Analyses of eBMD were performed on a sample of 362,924 unrelated white British subjects (54\% female, GCTA pairwise relatedness < 0.10 ) from the UK Biobank Study (UKB) ${ }^{118}$ that had valid quantitative eBMD and high-quality genome-wide HRC and $1000 \mathrm{G} / \mathrm{UK} 10 \mathrm{~K}$ imputed data from the January 2018 release [(i.e. 20,490,436 genetic variants with an information quality score $>0.3$, minor allele frequency $>0.05 \%$, minor allele count $>5$, genotyping hard call rate $>0.95$, and weak evidence of deviation from Hardy-Weinberg equilibrium $\left.\left(P>1 \times 10^{-6}\right)\right]$. Analyses of individuals with OA were performed on precomputed summary statistics from a recent UKBB and arcOGEN GWAS meta-analysis of four predefined OA subcategories: Osteoarthritis at any site (ALLOA): 77,052 cases and 378,169 controls, hip osteoarthritis (HIPOA): 15,704 cases and 378,169 controls, knee osteoarthritis (KNEEOA): 24,955 cases and 378169 controls and hip and/or knee (HIPKNEEOA): 39,427 cases and 378,169 controls. Details of how eBMD and $O A$ was defined and criteria for including individuals in each cohort are detailed in the original publications $s^{25,64}$. No human research participants were directly recruited for this work. Ethical approval for collection of data for the UKBiobank data was obtained from the Northwest Multi-Centre Research Ethics Committee and informed consent was obtained from all participants prior to participation. Project approval and access to the anonymised, deidentified UKBB data was granted with accession ID: 53641. The arcOGEN study was ethically approved by appropriate review committees, and the prospective collections were approved by the National Research Ethics Service in the United Kingdom. All subjects in arcOGEN provided written, informed consent.

Competitive gene set analysis. Overview: competitive gene set analysis involved a three-stage process. In the first stage mouse-human orthologues were mapped and the gene universe used for enrichment defined. In the second stage gene-based tests of association were performed using genome-wide genetic data to estimate the strength of association of each human protein-coding gene with eBMD or OA. In the third stage, competitive gene set analysis was used to compare the mean strength of association of human orthologues of osteocyte transcriptome signature genes, to the mean strength of association of non-osteocyte transcriptome signature genes. Evidence of enrichment was obtained through significance testing, in which the null hypothesis of no difference in mean association between osteocyte, and non-osteocyte signature gene sets was tested against a one-sided alternative that stipulated that osteocyte transcriptome signature genes were on average more strongly associated with eBMD or OA than non-osteocyte transcriptome signature genes.

Stage 1- orthologue mapping and universe definition: mouse-to-human orthologs were identified in the February 2014 Ensembl database archive, in line with the GRCh37 genome used in the GWAS analysis, accessed through the biomaRt R-package ${ }^{88}$. To ensure enrichment was calculated relative to genes that had a fair chance of identification in the osteocyte transcriptome signature, and avoid $P$-value inflation due to inclusion of genes not able to be assayed by RNAseq, the gene universe was limited to orthologs of genes actively expressed in any sample type from either of the Bone comparison, Skeletal maturation or Osteocyte enrichment cohorts, or any of the 12 non-skeletal tissues detailed in the Comparison with other organs and tissues section. This encompassed 16,015 mouse-human orthologs, including 992 of the Osteocyte transcriptome signature genes. All analyses were repeated relative to all human-mouse gene orthologs. In all cases, the results from analyses relative to the gene universe were more conservative than when all orthologs were used, and the more conservative results are presented here.

Stage 2- Gene-based tests of association: Gene-based tests of association were conducted in MAGMA (v1.06, https://ctg.cncr.nl/software/magma) ${ }^{62}$ using imputed individual level genotype data for the analyses involving eBMD, and GWAS meta-analysis summary results statistics for OA. Analyses involving eBMD were further adjusted for age, sex, genotyping array, assessment centre and ancestry informative principal components $1-20$. Gene-based tests of association encompassed a multi-model approach in which the association results from different gene analysis models were combined to produce an aggregate $\mathrm{p}$-value corresponding to the strength of evidence of association between each gene $( \pm 20$ $\mathrm{kb}$ ) and $\mathrm{eBMD}$ or $\mathrm{OA}$. The three association models included: a principal components regression model, a SNP-wise mean $\chi 2$ model [i.e. test statistic derived as the sum of $-\log ($ SNP $P$-value) for all SNPs that intersect the gene region of interest], and SNP-wise top $\chi^{2}$ model [(test statistic derived as the sum of $-\log$ (SNP $P$-value) for top SNP in the region of interest)]. The aggregate approach was chosen as it yields a more even distribution of statistical power and sensitivity over a wider range of different genetic architectures. Note: principal components regression model could not be run for $\mathrm{OA}$ as the method requires individual level genotyping data that was not available. The enrichment of genes with mutations known to cause human genetic skeletal disorders ${ }^{21}$ was examined under the hypergeometric distribution using the following parameters:

- Population: orthologs in the above defined gene universe (16,015 genes)

- Total number of successes in the population: genes in the population known to cause skeletal disorders when mutated in humans (432)

- Sample: osteocyte transcriptome signature orthologs in the population with significant gene-level associations with either $\mathrm{eBMD}$ or $\mathrm{OA}(\mathrm{eBMD}=259$, $\mathrm{OA}=40)$

- Number of sample successes: the number of Osteocyte transcriptome signature orthologs in the population with significant gene-level association with either eBMD or OA known to cause human skeletal disorders (eBMD = $36, \mathrm{OA}=8$ )

Significant enrichment was calculated using the Graeber lab online hypergeometric calculator (https://systems.crump.ucla.edu/hypergeometric/index. php).

Stage 3- Gene set analysis: competitive gene set analysis was used to determine whether the set of 992 osteocyte transcriptome signature human gene orthologues was on average more strongly associated with eBMD or OA than non-osteocyte signature genes. The analysis accounted for several confounding factors including: gene size, gene density (i.e. representing the relative level of LD between SNPs in the gene) and the inverse of the mean minor allele count in the gene (i.e. to correct for potential power loss in very low minor allele count SNPs), as well the log value of these three factors.

Human genome coordinates (hg19) were mapped and Circos plots generated for the osteocyte transcriptome signature orthologs with significant gene-level associations with $e B M D$ and $\mathrm{OA}$ using the ggbio R-package ${ }^{119}$. The top 100 genes associated with eBMD (ranked by $P$-value) were shown to avoid overplotting.

Stratified linkage disequilibrium score regression. LDSC-SEG ${ }^{60,61}$ was used in conjunction with summary results statistics from recent GWAS of eBMD and $\mathrm{OA}^{25,64}$ to investigate whether genomic regions surrounding osteocyte transcriptome signature human gene orthologs contribute disproportionately to the SNP heritability of eBMD and OA. Here, heritability was defined as the 
proportion of trait variation/disease liability explained by genome-wide imputed genetic markers and is referred to as SNP heritability. Enrichment is expressed in terms of per-SNP heritability, and estimated as the proportion of SNP heritability explained by genomic regions intersecting the gene-set, divided by the proportion of SNPs intersecting the corresponding gene set. Evidence of enrichment is evaluated though significance testing, in which the null hypothesis of no difference in per-SNP heritability, is tested against the one-sided alternative where the per-SNP attributable to the gene-set is greater than the per-SNP heritability attributable to the rest of genes in the genome. Using a similar approach to that described previously ${ }^{60,61}$, we constructed a genome-annotation for autosomal gene regions $\pm 20 \mathrm{~kb}$ of the osteocyte transcriptome signature orthologs, a second encompassing all mappable mouse-human genes, and a third annotation corresponding to the gene universe described above. We applied LDSC-SEG ${ }^{60,61}$ to jointly model the osteocyte transcriptome signature annotation, together with 52 functional genomic annotations that include genic regions, enhancer regions and conserved regions (i.e. baseline model vl.1 supplied with the LDSC-SEG software, https://github.com/bulik/ldsc). We limited the analysis to high-quality imputed autosomal SNPs (INFO $>0.9$ ), excluded the HLA region from all analyses and used the 1000 Genomes LD reference panel of unrelated European subjects as supplied with the software. Enrichment was quantified by the LDSC-SEG regression coefficient, which corresponds to the magnitude of enrichment in per-SNP eBMD heritability attributable to the osteocyte transcriptome signature, conditional on the gene universe and the 52 baseline annotations. Strength of evidence against the null hypothesis of no enrichment attributable to the osteocyte transcriptome signature annotation, conditional on other annotations was determined by the LDSC-SEG $P$-value. Sensitivity analysis was conducted by increasing the window to $\pm 100 \mathrm{~kb}$ of each gene and re-analysing.

Nearest-gene enrichment analysis. Lastly, we examined whether osteocyte transcriptome signature orthologs were located nearest to genome wide significant (GWS), conditionally independent GWAS loci associated with eBMD and OA more often than would be expected by chance. This was examined under the hypergeometric distribution using the following parameters:

- Population: orthologs in the above defined gene universe (16,015 genes)

- Total number of successes in the population: number of osteocyte transcriptome signature orthologs in the population (992)

- Sample: genes in the population that are the nearest gene to a GWS loci $(\mathrm{eBMD}=638, \mathrm{OA}=52)$

- Number of sample successes: number of osteocyte transcriptome signature orthologs in the population that are the nearest gene to a GWS loci (eBMD = $110, \mathrm{OA}=13$ )

Significant enrichment was calculated using the Graeber lab online hypergeometric calculator (https://systems.crump.ucla.edu/hypergeometric/index. php). Only unique genes were used to calculate enrichment (genes located nearest to multiple GWS loci were only counted once).

Quantification and statistical analysis. Statistical methodologies and software used for performing these analyses are described in the appropriate sections. Biological replicates were taken from distinct samples. Analyses using Trinity (v2.0.6), Stringtie (v1.0.4), Cuffcompare (v2.2.1), Trimgalore (v0.3.3), STAR (v2.4.1d) and RSEM (v1.2.21) were performed on a computing cluster running the CentOS 6.8 (Rocks 6.2) Linux operating system. CTAn, NRecon and Drishti were run using a Windows 7 OS. CPAT and ReViGo analyses were run using the web interface. Hypergeometric enrichment was tested using the Graeber lab online hypergeometric calculator (https://systems.crump.ucla.edu/hypergeometric/index. php). Gene-based tests of association and competitive gene set analysis were conducted with MAGMA (v1.06, https://ctg.cncr.nl/software/magma). LDSC-SEG was conducted with LDSC-SEG software, https://github.com/bulik/ldsc (v1.1). All other statistical analysis was performed in R ( > v3.4.0), with key packages cited in the methods text. Error bars reflect mean and standard deviation unless stated otherwise. Multiple hypothesis correction was used wherever significance was evaluated across multiple statistical tests i.e. differential gene expression analysis (Benjamini-Hochberg FDR), GO enrichment, KEGG enrichment, DO enrichment, Nosology group enrichment (Bonferroni correction).

Reporting summary. Further information on research design is available in the Nature Research Reporting Summary linked to this article.

\section{Data availability}

The raw RNA-sequencing data (fastq), read alignment files (BAM) and processed gene expression data files for each cohort (FPKM and counts) are deposited at ArrayExpress (https://www.ebi.ac.uk/arrayexpress) under the following accession numbers: bone comparison cohort (E-MTAB-5532, https://www.ebi.ac.uk/arrayexpress/experiments/EMTAB-5532), skeletal maturation cohort (E-MTAB-7447, https://www.ebi.ac.uk/ arrayexpress/experiments/E-MTAB-7447) and osteocyte enrichment cohort (E-MTAB5533, https://www.ebi.ac.uk/arrayexpress/experiments/E-MTAB-5533). There are no restrictions on data availability. Various publicly available data resources were used in this study: To compare the osteocyte transcriptome to that of other tissues, publicly available sequencing read data was obtained (ArrayExpress accession E-GEOD-54652, https://www.ebi.ac.uk/arrayexpress/experiments/E-GEOD-54652). To determine whether the Magenta Cluster identified genes associated with perilacunar- remodelling their expression was examined during lactation using a publicly available microarray dataset (ArrayExpress accession E-GEOD-23496, https://www.ebi.ac.uk/arrayexpress/ experiments/E-GEOD-23496). To compare expression of osteocyte transcriptome signature genes in osteocyte with other bone-cell types we used a publicly available microarray dataset which profiled gene expression in laser-capture micro-dissected osteoblasts, bone-lining cells and osteocytes (GEO accession GSE71306, https://www. ncbi.nlm.nih.gov/geo/query/acc.cgi?acc=GSE71306). To examine the expression of osteocyte transcriptome signature genes during osteocyte differentiation, we analysed publicly available transcriptome sequencing data from an in vitro model of osteoblastlike cell to osteocyte differentiation (ArrayExpress accession E-GEOD-54783, https:// www.ebi.ac.uk/arrayexpress/experiments/E-GEOD-54783). Human genotype and phenotype data on which the results of this study were based were accessed from the UK Biobank (http://www.ukbiobank.ac.uk/) with accession ID 53641.

\section{Code availability}

Custom code used to define gene activity, gene expression enrichment in osteocytes and the osteocyte transcriptome signature are available in a public Github repository accessible at https://github.com/scottyoulten/osteocyte_signature.

Received: 3 April 2020; Accepted: 11 March 2021; Published online: 05 May 2021

\section{References}

1. Dallas, S. L., Prideaux, M. \& Bonewald, L. F. The osteocyte: an endocrine cell... and more. Endocr. Rev. 34, 658-690 (2013).

2. Kollmannsberger, P. et al. The small world of osteocytes: connectomics of the lacuno-canalicular network in bone. New J. Phys. 19, 073019 (2017).

3. Buenzli, P. R. \& Sims, N. A. Quantifying the osteocyte network in the human skeleton. Bone 75, 144-150 (2015).

4. Schaffler, M. B., Cheung, W. Y., Majeska, R. \& Kennedy, O. Osteocytes: master orchestrators of bone. Calcif. Tissue Int. 94, 5-24 (2014).

5. Bergwitz, C. \& Jüppner, H. Regulation of phosphate homeostasis by PTH, vitamin D, and FGF23. Annu. Rev. Med. 61, 91-104 (2010).

6. Sato, M. et al. Osteocytes regulate primary lymphoid organs and fat metabolism. Cell Metab. 18, 749-758 (2013).

7. Asada, N. et al. Matrix-embedded osteocytes regulate mobilization of hematopoietic stem/progenitor cells. Cell Stem Cell 12, 737-747 (2013).

8. Shen, H., Grimston, S., Civitelli, R. \& Thomopoulos, S. Deletion of connexin43 in osteoblasts / osteocytes leads to impaired muscle formation in mice. J. Bone Miner. Res. 30, 596-605 (2015).

9. Forrest, A. R. R. R. et al. A promoter-level mammalian expression atlas. Nature 507, 462-470 (2014).

10. Lonsdale, J. et al. The genotype-tissue expression (GTEx) project. Nat. Genet. 45, 580-585 (2013).

11. Petryszak, R. et al. Expression Atlas update - an integrated database of gene and protein expression in humans, animals and plants. Nucleic Acids Res. 44, 746-752 (2016).

12. The Tabula Muris Consortium et al. Single-cell transcriptomics of 20 mouse organs creates a Tabula Muris. Nature https://doi.org/10.1038/s41586-0180590-4 (2018)

13. Ayturk, U. M. et al. An RNA-seq protocol to identify mRNA expression changes in mouse diaphyseal bone: applications in mice with bone property altering Lrp5 mutations. J. Bone Miner. Res. 28, 2081-2093 (2013).

14. Kelly, N. H., Schimenti, J. C., Ross, F. P. \& van der Meulen, M. C. H. Transcriptional profiling of cortical versus cancellous bone from mechanically-loaded murine tibiae reveals differential gene expression. Bone 86, 22-29 (2016)

15. Qing, H. et al. Demonstration of osteocytic perilacunar/canalicular remodeling in mice during lactation. J. Bone Miner. Res. 27, 1018-1029 (2012).

16. Nioi, P. et al. Transcriptional profiling of laser capture microdissected subpopulations of the osteoblast lineage provides insight into the early response to sclerostin antibody in rats. J. Bone Miner. Res. 30, 1457-1467 (2015).

17. Brunkow, M. E. et al. Bone dysplasia sclerosteosis results from loss of the SOST gene product, a novel cystine knot-containing protein. Am. J. Hum. Genet. 68, 577-589 (2001).

18. Balemans, W. et al. Increased bone density in sclerosteosis is due to the deficiency of a novel secreted protein (SOST). Hum. Mol. Genet. 10, 537-543 (2001). 
19. Balemans, W. et al. Identification of a $52 \mathrm{~kb}$ deletion downstream of the SOST gene in patients with van Buchem disease. J. Med. Genet. 39, 91-97 (2002).

20. Feng, J. Q. et al. Loss of DMP1 causes rickets and osteomalacia and identifies a role for osteocytes in mineral metabolism. Nat. Genet. 38, 1310-1315 (2006).

21. Mortier, G. R. et al. Nosology and classification of genetic skeletal disorders: 2019 revision. Am. J. Med. Genet. A https://doi.org/10.1002/ajmg.a.61366 (2019).

22. Videman, T. et al. Heritability of BMD of femoral neck and lumbar spine: a multivariate twin study of Finnish men. J. Bone Miner. Res. 22, 1455-1462 (2007).

23. Karasik, D. et al. Mapping of quantitative ultrasound of the calcaneus bone to chromosome 1 by genome-wide linkage analysis. Osteoporos. Int. 13, 796-802 (2002).

24. Valdes, A. M. \& Spector, T. D. Genetic epidemiology of hip and knee osteoarthritis. Nat. Rev. Rheumatol. 7, 23-32 (2011).

25. Morris, J. A. et al. An atlas of genetic influences on osteoporosis in humans and mice. Nat. Genet. 51, 258-266 (2019).

26. Zengini, E. et al. Genome-wide analyses using UK Biobank data provide insights into the genetic architecture of osteoarthritis. Nat. Genet. 50, 549-558 (2018).

27. Hart, T., Komori, H. K., LaMere, S., Podshivalova, K. \& Salomon, D. R. Finding the active genes in deep RNA-seq gene expression studies. BMC Genomics 14, 778 (2013).

28. Zhang, R., Lahens, N. F., Ballance, H. I., Hughes, M. E. \& Hogenesch, J. B. A circadian gene expression atlas in mammals: Implications for biology and medicine. Proc. Natl Acad. Sci. USA 111, 16219-16224 (2014).

29. Yanai, I. et al. Genome-wide midrange transcription profiles reveal expression level relationships in human tissue specification. Bioinformatics 21, 650-659 (2005).

30. Agarwal, P. et al. Tbx 5 is essential for forelimb bud initiation following patterning of the limb field in the mouse embryo. Development 130, 623-633 (2003).

31. Takeuchi, J. K. et al. Tbx5 and Tbx4 genes determine the wing/leg identity of limb buds. Nature 398, 810-814 (1999).

32. DeLaurier, A., Schweitzer, R. \& Logan, M. Pitxl determines the morphology of muscle, tendon, and bones of the hindlimb. Dev. Biol. 299, 22-34 (2006).

33. Spielmann, M. et al. Homeotic arm-to-leg transformation associated with genomic rearrangements at the PITX1 locus. Am. J. Hum. Genet. 91, 629-635 (2012).

34. Richman, C. et al. Postnatal and pubertal skeletal changes contribute predominantly to the differences in peak bone density between $\mathrm{C} 3 \mathrm{H} / \mathrm{HeJ}$ and C57BL/6J mice. J. Bone Miner. Res. 16, 386-397 (2001).

35. Cole, T. J. et al. Ethnic and sex differences in skeletal maturation among the Birth to Twenty cohort in South Africa. Arch. Dis. Child. 100, 138-143 (2015).

36. Baxter-Jones, A. D. G., Faulkner, R. A., Forwood, M. R., Mirwald, R. L. \& Bailey, D. A. Bone mineral accrual from 8 to 30 years of age: An estimation of peak bone mass. J. Bone Miner. Res. 26, 1729-1739 (2011).

37. Jähn, K. et al. Osteocytes acidify their microenvironment in response to PTHrP in vitro and in lactating mice in vivo. J. Bone Miner. Res. 32, 1761-1772 (2017).

38. Plotkin, L. I. \& Bellido, T. Osteocytic signalling pathways as therapeutic targets for bone fragility. Nat. Rev. Endocrinol. 12, 593-605 (2016).

39. Taylor, S. et al. Differential time-dependent transcriptional changes in the osteoblast lineage in cortical bone associated with sclerostin antibody treatment in ovariectomized rats. Bone Rep. 8, 95-103 (2018).

40. Ashburner, M. et al. Gene ontology: tool for the unification of biology. Nat. Genet. 25, 25-29 (2000).

41. Smith, C. L., Blake, J. A., Kadin, J. A., Richardson, J. E. \& Bult, C. J. Mouse Genome Database (MGD)-2018: knowledgebase for the laboratory mouse. Nucleic Acids Res. 46, D836-D842 (2018).

42. Joeng, K. S. et al. Osteocyte-specific WNT1 regulates osteoblast function during bone homeostasis. J. Clin. Invest. 127, 2678-2688 (2017).

43. Wang, L. et al. Osteoblast-derived FGF9 regulates skeletal homeostasis. Bone 98, 18-25 (2017)

44. Bai, M. et al. Targeted genetic screening in mice through haploid embryonic stem cells identifies critical genes in bone development. PLoS Biol. 17, e3000350 (2019)

45. Han, Q. et al. 4H-Thieno[3,2-c]chromene based inhibitors of Notum Pectinacetylesterase. Bioorg. Med. Chem. Lett. 26, 1184-1187 (2016).

46. Brommage, R. et al. NOTUM inhibition increases endocortical bone formation and bone strength. Bone Res. 7, 2 (2019).

47. Movérare-Skrtic, S. et al. Osteoblast-derived NOTUM reduces cortical bone mass in mice and the NOTUM locus is associated with bone mineral density in humans. FASEB J. 33, 11163-11179 (2019).
48. Goff, C. L. E. et al. ADAMTSL2 mutations in geleophysic dysplasia demonstrate a role for ADAMTS-like proteins in TGF- $\beta$ bioavailability regulation. Nat. Genet. 40, 1119-1123 (2008).

49. Stoeckli, E. T. Understanding axon guidance: are we nearly there yet? Development 145, dev151415 (2018).

50. St. John, H. C. et al. The osteoblast to osteocyte transition: epigenetic changes and response to the vitamin $\mathrm{D}_{3}$ hormone. Mol. Endocrinol. 28, 1150-1165 (2014).

51. Balasubramanian, M. et al. Novel PLS3 variants in X-linked osteoporosis: exploring bone material properties. Am. J. Med. Genet. A 176, 1578-1586 (2018).

52. Hori, K. et al. Cytoskeletal regulation by AUTS2 in neuronal migration and neuritogenesis. Cell Rep. 9, 2166-2179 (2014).

53. Jiang, $X$. et al. DACT3 is an epigenetic regulator of wnt/ $\beta$-catenin signaling in colorectal cancer and is a therapeutic target of histone modifications. Cancer Cell 13, 529-541 (2008).

54. Lee, H. K. \& Deneen, B. Daam2 is required for dorsal patterning via modulation of canonical wnt signaling in the developing spinal cord. Dev. Cell 22, 183-196 (2012)

55. Nakano, N. et al. C18 orf1, a novel negative regulator of transforming growth factor- $\beta$ signaling. J. Biol. Chem. 289, 12680-12692 (2014).

56. Chen, Y. K. \& Hsueh, Y. P. Cortactin-binding protein 2 modulates the mobility of cortactin and regulates dendritic spine formation and maintenance. J. Neurosci. 32, 1043-1055 (2012).

57. Marini, J. C. et al. Osteogenesis imperfecta. Nat. Rev. Dis. Primers. 3, 17052 (2017).

58. Doyard, M. et al. FAM46A mutations are responsible for autosomal recessive osteogenesis imperfecta. J. Med. Genet. 55, 278-284 (2018).

59. Garbes, L. et al. Mutations in SEC24D, encoding a component of the COPII machinery, cause a syndromic form of osteogenesis imperfecta. Am. J. Hum. Genet. 96, 432-439 (2015).

60. Finucane, H. K. et al. Partitioning heritability by functional annotation using genome-wide association summary statistics. Nat. Genet. 47, 1228-1235 (2015).

61. Bulik-Sullivan, B. et al. LD score regression distinguishes confounding from polygenicity in genome-wide association studies. Nat. Genet. 47, 291-295 (2015).

62. de Leeuw, C. A., Mooij, J. M., Heskes, T. \& Posthuma, D. MAGMA: generalized gene-set analysis of GWAS data. PLoS Comput. Biol. 11, 1-19 (2015).

63. Glyn-Jones, S. et al. Osteoarthritis. Lancet 386, 376-387 (2015).

64. Tachmazidou, I. et al. Identification of new therapeutic targets for osteoarthritis through genome-wide analyses of UK Biobank data. Nat. Genet. 51, 230-236 (2019).

65. Sanchez, C. et al. Chondrocyte secretome: a source of novel insights and exploratory biomarkers of osteoarthritis. Osteoarthritis Cartilage 25, 1199-1209 (2017).

66. Bassett, J. H. D. et al. Rapid-throughput skeletal phenotyping of 100 knockout mice identifies 9 new genes that determine bone strength. PLoS Genet. 8, e1002858 (2012).

67. Leucht, P. et al. Embryonic origin and Hox status determine progenitor cel fate during adult bone regeneration. Development 135, 2845-2854 (2008).

68. Rux, D. R. et al. Regionally restricted hox function in adult bone marrow multipotent mesenchymal stem/stromal cells. Dev. Cell 39, 653-666 (2016).

69. Karam, N. et al. Bone-specific overexpression of PITX1 induces senile osteoporosis in mice through deficient self-renewal of mesenchymal progenitors and wnt pathway inhibition. Sci. Rep. 9, 1-15 (2019).

70. Fowler, T. W. et al. Glucocorticoid suppression of osteocyte perilacunar remodeling is associated with subchondral bone degeneration in osteonecrosis. Sci. Rep. 7, 1-13 (2017).

71. Goldring, S. R. \& Goldring, M. B. Changes in the osteochondral unit during osteoarthritis: Structure, function and cartilage bone crosstalk. Nat. Rev. Rheumatol. 12, 632-644 (2016).

72. Blouin, S. et al. Hypermineralization and high osteocyte lacunar density in osteogenesis imperfecta type $\mathrm{V}$ bone indicate exuberant primary bone formation. J. Bone Miner. Res. 32, 1884-1892 (2017).

73. Zimmerman, S. M., Dimori, M., Heard-Lipsmeyer, M. E. \& Morello, R. The osteocyte transcriptome is extensively dysregulated in mouse models of osteogenesis imperfecta. JBMR Plus 3, e10171 (2019).

74. Storheim, K. \& Zwart, J. A. Musculoskeletal disorders and the Global Burden of Disease study. Ann. Rheum. Dis. https://doi.org/10.1136/annrheumdis2014-205327 (2014)

75. Limaye, A. Drishti: A Volume Exploration And Presentation Tool. Vol. 8506 (SPIE Proceedings, 2012).

76. Schindelin, J. et al. Fiji: an open-source platform for biological-image analysis. Nat. Methods 9, 676-682 (2012).

77. Adiconis, X. et al. Comparative analysis of RNA sequencing methods for degraded or low-input samples. Nat. Methods 10, 623-629 (2013). 
78. Haas, B. J. et al. De novo transcript sequence reconstruction from RNA-seq using the Trinity platform for reference generation and analysis. Nat. Protoc. 8, 1494-1512 (2013).

79. Pertea, M. et al. StringTie enables improved reconstruction of a transcriptome from RNA-seq reads. Nat. Biotechnol. 33, 290-295 (2015).

80. Trapnell, C. et al. Differential gene and transcript expression analysis of RNAseq experiments with TopHat and Cufflinks. Nat. Protoc. 7, 562-578 (2012).

81. Pruitt, K. D. et al. RefSeq: an update on mammalian reference sequences. Nucleic Acids Res. 42, 756-763 (2014).

82. Mudge, J. M. \& Harrow, J. Creating reference gene annotation for the mouse C57BL6/J genome assembly. Mamm. Genome 26, 366-378 (2015).

83. Wang, L. et al. CPAT: coding-potential assessment tool using an alignmentfree logistic regression model. Nucleic Acids Res. 41, 1-7 (2013).

84. Dobin, A. et al. STAR: ultrafast universal RNA-seq aligner. Bioinformatics 29, 15-21 (2013).

85. Li, B. \& Dewey, C. N. RSEM: accurate transcript quantification from RNASeq data with or without a reference genome. BMC Bioinform. 12, 323 (2011).

86. Woo, S. M., Rosser, J., Dusevich, V., Kalajzic, I. \& Bonewald, L. F. Cell line IDG-SW3 replicates osteoblast-to-late-osteocyte differentiation in vitro and accelerates bone formation in vivo. J. Bone Miner. Res. 26, 2634-2646 (2011).

87. Taylor, S. et al. Time-dependent cellular and transcriptional changes in the osteoblast lineage associated with sclerostin antibody treatment in ovariectomized rats. Bone 84, 148-159 (2016).

88. Durinck, S., Spellman, P. T., Birney, E. \& Huber, W. Mapping identifiers for the integration of genomic datasets with the $\mathrm{R} /$ Bioconductor package biomaRt. Nat. Protoc. 4, 1184-1191 (2009).

89. MacDonald, J. W. affycoretools: functions useful for those doing repetitive analyses with Affymetrix GeneChips. $R$ Packag. version 1, (2008).

90. Carvalho, B. S. \& Irizarry, R. A. A framework for oligonucleotide microarray preprocessing. Bioinformatics 26, 2363-2367 (2010).

91. Gautier, L., Cope, L., Bolstad, B. M. \& Irizarry, R. A. Affy - Analysis of Affymetrix GeneChip data at the probe level. Bioinformatics 20, 307-315 (2004).

92. Wickham, H. ggplot2: elegant graphics for data analysis. (Springer, 2016).

93. Ritchie, M. E. et al. Limma powers differential expression analyses for RNAsequencing and microarray studies. Nucleic Acids Res. 43, e47 (2015)

94. Law, C. W., Chen, Y., Shi, W. \& Smyth, G. K. Voom: Precision weights unlock linear model analysis tools for RNA-seq read counts. Genome Biol. 15, 1-17 (2014).

95. Langfelder, P. \& Horvath, S. WGCNA: an R package for weighted correlation network analysis. BMC Bioinform. 9, 559 (2008).

96. Langfelder, P., Zhang, B. \& Horvath, S. Defining clusters from a hierarchical cluster tree: The Dynamic Tree Cut package for R. Bioinformatics 24, 719-720 (2008).

97. Ogata, H. et al. KEGG: Kyoto encyclopedia of genes and genomes. Nucleic Acids Res. 27, 29-34 (1999).

98. Kibbe, W. A. et al. Disease Ontology 2015 update: an expanded and updated database of human diseases for linking biomedical knowledge through disease data. Nucleic Acids Res. 43, 1071-1078 (2018).

99. Yu, G., Wang, L.-G., Han, Y. \& He, Q.-Y. clusterProfiler: an R package for comparing biological themes among gene clusters. OMICS 16, 284-287 (2012).

100. Yu, G., Wang, L. G., Yan, G. R. \& He, Q. Y. DOSE: an R/Bioconductor package for disease ontology semantic and enrichment analysis. Bioinformatics 31, 608-609 (2015).

101. Warnes, G. R. et al. gplots: various R programming tools for plotting data. $R$. Package version 2, 1 (2009).

102. Supek, F., Bošnjak, M., Škunca, N. \& Šmuc, T. Revigo summarizes and visualizes long lists of gene ontology terms. PLOS ONE 6, e21800 (2011).

103. Fraley, C. \& Raftery, A. E. Enhanced model-based clustering, density estimation, and discriminant analysis software: MCLUST. J. Classif. 20, 263-286 (2003).

104. Wu, D. \& Smyth, G. K. Camera: a competitive gene set test accounting for inter-gene correlation. Nucleic Acids Res. 40, 1-12 (2012).

105. Benaglia, T., Chauveau, D., Hunter, D. \& Young, D. mixtools: an R package for analyzing finite mixture models. J. Stat. Softw. 32, 1-29 (2009).

106. Youlten, S. E. \& Baldock, P. A. Using mouse genetics to understand human skeletal disease. Bone 126, 27-36 (2019).

107. Luo, W. \& Brouwer, C. Pathview: an R/Bioconductor package for pathwaybased data integration and visualization. Bioinformatics 29, 1830-1831 (2013).

108. Wang, X., Qiu, W. \& Zamar, R. H. CLUES: a non-parametric clustering method based on local shrinking. Comput. Stat. Data Analysis 52, 286-298 (2007).

109. Hahne, F. \& Ivanek, R. in Statistical Genomics p. 335-351 (Springer, 2016)

110. Yang, H., Wang, H. \& Jaenisch, R. Generating genetically modified mice using CRISPR/Cas-mediated genome engineering. Nat. Protoc. 9, 1956-1968 (2014).
111. International, T. \& Knockout, M. A mouse for all reasons. Cell 128, 9-13 (2007).

112. Koscielny, G. et al. The International Mouse Phenotyping Consortium Web Portal, a unified point of access for knockout mice and related phenotyping data. Nucleic Acids Res. 42, 802-809 (2014).

113. Skarnes, W. C. et al. A conditional knockout resource for the genome-wide study of mouse gene function. Nature 474, 337-344 (2011).

114. Boroviak, K., Doe, B., Banerjee, R., Yang, F. \& Bradley, A. Chromosome engineering in zygotes with CRISPR/Cas9. Genesis 54, 78-85 (2016).

115. De Angelis, M. H. et al. Analysis of mammalian gene function through broadbased phenotypic screens across a consortium of mouse clinics. Nat. Genet. 47, 969-978 (2015).

116. Doube, M. et al. BoneJ: free and extensible bone image analysis in ImageJ. Bone 47, 1076-1079 (2010).

117. Ollion, J., Cochennec, J., Loll, F., Escudé, C. \& Boudier, T. TANGO: a generic tool for high-throughput 3D image analysis for studying nuclear organization. Bioinformatics 29, 1840-1841 (2013).

118. Bycroft, C. et al. The UK Biobank resource with deep phenotyping and genomic data. Nature 562, 203-209 (2018).

119. Yin, T., Cook, D. \& Lawrence, M. ggbio: an R package for extending the grammar of graphics for genomic data. Genome Biol. 13, R77 (2012).

\section{Acknowledgements}

This work was supported by a Wellcome Trust Strategic Award (101123/Z/13/A) to G.R. W, P.I.C. and J.H.D.B, and by Mrs Janice Gibson and the Ernest Heine Family Foundation. P.I.C is supported by Mrs Janice Gibson and the Ernest Heine Family Foundation. J.H.D.B and G.R.W received a Wellcome Trust Joint Investigator Award (110141/Z/ 15/Z and 110140/Z/15/Z) and EU H2020 THYRAGE Grant (666869). S.E.Y and A.P.C were funded by the Australian Government Research Training Program Scholarships. J. P.K was funded by a University of Queensland Development Fellowship (UQFEL1718945), a National Health and Medical Research Council (Australia) Investigator grant (GNT1177938) and project grant (GNT1158758). J.T.S was funded by a University International Postgraduate Award Scholarship (UNSW). J.A.M was funded by a Banting Postdoctoral Fellowship. J.M.W.Q was funded by an Australian National Health and Medical Research Council project grant (ID: GNT1057706). M.J was funded by Viapath. J.B.R was supported by the Canadian Institutes of Health Research (CIHR), the Lady Davis Institute of the Jewish General Hospital, the Canadian Foundation for Innovation, the NIH Foundation, Cancer Research UK and the Fonds de Recherche Québec Santé (FRQS). J.B.R. is supported by a FRQS Clinical Research Scholarship. TwinsUK is funded by the Welcome Trust, Medical Research Council, European Union, the National Institute for Health Research (NIHR)-funded BioResource, Clinical Research Facility and Biomedical Research Centre based at Guy's and St Thomas' NHS Foundation Trust in partnership with King's College London. D.J.A and C.J.L were funded by Wellcome Trust Strategic Awards. D.M.E. was funded by an Australian National Health and Medical Research Council Senior Research Fellowship (APP1137714). T.G.P was funded by an Australian National Health and Medical Research Council Senior Research Fellowship (1155678). This research has been conducted using the UK Biobank Resource (accession IDs: 12703). This work was assisted by phenotyping expertise provided by Anne-Tounsia Adoum, Justyna Miszkiewicz, Rebecca Allen, Jayashree Bagchi Chakraborty, Katharine F Curry, Hannah F. Dewhurst, Apostolos Gogakos, Naila Mannan, Hayley J Protheroe, Penny C Sparkes, Valerie Vancollie and Fiona Kussy. Ya Xiao assisted with animal studies. The Sanger Institute's Research Support Facility, Mouse Pipelines and Mouse Informatics Group generated mice and collected materials for this manuscript. This research has been conducted using the UK Biobank Resource (accession ID: 53641).

\section{Author contributions}

S.E.Y., J.H.D.B., G.R.W. and P.I.C. conceived the study. S.E.Y., J.P.K., M.I., A.B-M., F.R., E.D., J.B.R., R.B., T.G.P., J.A.E., D.M.E., E.Z., P.A.B., J.H.D.B., G.R.W. and P.I.C. designed experiments. S.E.Y., J.G.L., E.J.G., C.M.S., M.D., S.E.G., V.D.L., N.C.B., D.K.-E., R.C.C., S. T.M., M.M.M., J.M.W.Q. and A.R.M. conducted experiments. J.G.L., E.J.G., C.M.S., S.T M., M.R.G.D., S.E.G., V.D.L., N.C.B., D.K.-E., R.C.C., D.J.A., C.J.L. and R.B. generated mouse models and/or functional experiments. S.E.Y., J.P.K., J.G.L., C.M.S., S.T.M., M.I. D., S.E.G., V.D.L., N.C.B., D.K.-E., R.C.C., A.P.C., J.T.S., J.A.M., N.B., M.J., K.H., J.H.D.B., G.R.W. and P.I.C. performed data analysis. S.E.Y. was the lead analyst. S.E.Y., J.P.K., J.H. D.B., G.R.W. and P.I.C. wrote the manuscript. All authors revised and reviewed the paper.

\section{Competing interests}

T.G.P. is a consultant for Imugene Pty Ltd, an Australian biotech working in cancer immunotherapy. P.I.C. has funding from AMGEN for studies of cancer dormancy. Neither competing interests are the subject of this manuscript. The other authors declare no competing interests. 


\section{Additional information}

Supplementary information The online version contains supplementary material available at https://doi.org/10.1038/s41467-021-22517-1.

Correspondence and requests for materials should be addressed to J.H.D.B., G.R.W. or P.I.C.

Peer review information: Nature Communications thanks Roy Morello, Sundeep Khosla and the other, anonymous, reviewer(s) for their contribution to the peer review of this work.

Reprints and permission information is available at http://www.nature.com/reprints

Publisher's note Springer Nature remains neutral with regard to jurisdictional claims in published maps and institutional affiliations. (c) (i) Open Access This article is licensed under a Creative Commons Attribution 4.0 International License, which permits use, sharing, adaptation, distribution and reproduction in any medium or format, as long as you give appropriate credit to the original author(s) and the source, provide a link to the Creative Commons license, and indicate if changes were made. The images or other third party material in this article are included in the article's Creative Commons license, unless indicated otherwise in a credit line to the material. If material is not included in the article's Creative Commons license and your intended use is not permitted by statutory regulation or exceeds the permitted use, you will need to obtain permission directly from the copyright holder. To view a copy of this license, visit http://creativecommons.org/ licenses/by/4.0/.

(C) The Author(s) 2021

${ }^{1}$ Bone Biology, Garvan Institute of Medical Research, Darlinghurst, Sydney, NSW, Australia. ${ }^{2}$ St Vincent's Clinical School, Faculty of Medicine, UNSW Sydney, Sydney, NSW, Australia. ${ }^{3}$ University of Queensland Diamantina Institute, UQ, Brisbane, QLD, Australia. ${ }^{4} \mathrm{MRC}$ Integrative Epidemiology Unit, University of Bristol, Bristol, UK. ${ }^{5}$ Molecular Endocrinology Laboratory, Department of Metabolism, Digestion and Reproduction, Imperial College London, London, UK. ${ }^{6}$ RMIT Centre for Additive Manufacturing, School of Engineering, RMIT University, Melbourne, VIC, UK. ${ }^{7}$ Faculty of Science, University of Bath, Bath, UK. ${ }^{8}$ New York Genome Center, New York, NY, USA. ${ }^{9}$ Faculty of Arts and Science, Department of Biology, New York University, New York, NY, USA. ${ }^{10}$ Garvan Institute of Medical Research and The Kinghorn Cancer Centre, Darlinghurst, Sydney, NSW, Australia. ${ }^{11}$ Viapath Genetics Laboratory, Viapath Analytics LLP, Guy's Hospital, London, UK. ${ }^{12}$ Department of Clinical Genetics, Guy's Hospital, London, UK. ${ }^{13}$ Institute of Translational Genomics, Helmholtz Zentrum München - German Research Center for Environmental Health, Phoenix, AZ, USA. ${ }^{14}$ Wellcome Sanger Institute, Wellcome Genome Campus, Hinxton, UK. ${ }^{15}$ Department of Clinical Genetics, Guy's and St Thomas' NHS Trust, London, UK. ${ }^{16}$ Department of Clinical Genetics, University Hospitals Bristol, Bristol, UK. ${ }^{17}$ Department of Internal Medicine, Erasmus, MC, Rotterdam, the Netherlands. ${ }^{18}$ Faculty of Life Sciences and Medicine, Department of Twin Research \& Genetic Epidemiology, School of Life Course Sciences, King's College London, London, UK. ${ }^{19}$ Australian Translational Genomics Centre, Institute of Health and Biomedical Innovation, Faculty of Health, Queensland University of Technology, Brisbane, QLD, Australia. ${ }^{20}$ Faculty of Medicine, University of Queensland, St Lucia, QLD, Australia. ${ }^{21}$ Faculty of Medicine, McGill University, Quebec, Canada. ${ }^{22}$ Wellcome Sanger Institute, Hinxton, UK. ${ }^{23}$ Division of Immunology, Garvan Institute of Medical Research, Darlinghurst, Sydney, NSW, Australia. ${ }^{24}$ School of Medicine Sydney, University of Notre Dame Australia, Fremantle, Australia. ${ }^{25}$ School of Biotechnology and Biomolecular Sciences, UNSW Australia, Sydney, Australia. ${ }^{26}$ These

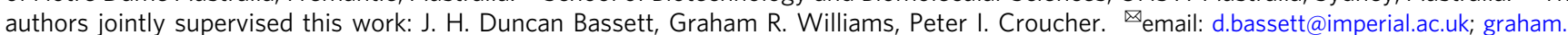
williams@imperial.ac.uk; p.croucher@garvan.org.au 